\title{
Small Nucleolar RNA and Small Nucleolar RNA Host Gene Signatures as Biomarkers for Pancreatic Cancer
}

\author{
Shuwen Han \\ Huzhou Central Hospital \\ Yangyang Xie \\ Ningbo No 2 Hospital \\ Xi Yang \\ Huzhou Central Hospital
}

\section{Siqi Dai}

Zhejiang University School of Medicine Second Affiliated Hospital

Xiaoyu Dai ( $\sim$ daixiaoyu@ucas.ac.cn )

University of the Chinese Academy of Sciences https://orcid.org/0000-0001-7276-5190

\section{Research}

Keywords: Pancreatic cancer, Small nucleolar noncoding RNAs, Small nucleolar RNA host gene, Biomarker, CeRNA network, Functional enrichment analysis

Posted Date: July 20th, 2020

DOI: https://doi.org/10.21203/rs.3.rs-42719/v1

License: (c) (i) This work is licensed under a Creative Commons Attribution 4.0 International License. Read Full License 


\section{Abstract}

Objective: The objective of this study was to identify key molecules including small nucleolar RNAs (snoRNAs) and small nucleolar RNA host genes (SNHGs) involved in pancreatic cancer.

Methods: First, we screened the differentially expressed snoRNAs (DEsnoRNAs) and trend-related snoRNAs based on the cancer genome atlas (TCGA) dataset for pancreatic cancer, and then performed methylation correlation analysis, survival analysis, and extraction of snoRNA host genes for Gene ontology (GO) functional and the Kyoto Encyclopedia of Genes and Genomes (KEGG) pathway enrichment analysis. Next, DESNHGs and trend-related SNHGs were screened according to the TCGA dataset for pancreatic cancer, and a competing endogenous RNA (ceRNA) network was constructed for pathway and functional enrichment analysis.

Results: A total of eight DEsnoRNAs and 93 trend-related snoRNAs were extracted. Then, ten host genes of the snoRNAs were identified. Functional analysis suggested that the ten host genes were significantly enriched in several GO terms including mitotic chromosome condensation and endocytosis pathway. SNORA38B was considered to associate with survival and prognosis. The SNORD17 and SNORA11 were considered to negatively correlate with methylation. In addition, two trend-related SNHGs were extracted. Additionally, a ceRNA network was constructed with 11 miRNAs, one IncRNAs, and one mRNA. SNHG24 mainly correlated with GnRH secretion and neuroactive ligand-receptor interaction in pancreatic cancer.

Conclusion: The identified snoRNAs and SNHGs could serve as potential markers for the early detection of pancreatic cancer.

\section{Introduction}

Pancreatic cancer is one of the most common malignancies of the digestive system. It has a poor prognosis due to its short disease course, high degree of malignancy, and challenges in early detection and treatment [1]. According to statistics, only about $24 \%$ of the patients survive for one year, and $9 \%$ of the patients survive for more than five years following the diagnosis of pancreatic cancer [2]. According to GLOBOCAN (Global cancer epidemic statistics) estimates, 458,918 new cases of pancreatic cancer were diagnosed in 2018 (accounting for $2.5 \%$ of all cancers), that resulted in 438,242 deaths, accounting for $4.5 \%$ of all cancer-related deaths [3]. At present, due to the low efficiency of surgical resection and chemotherapy, and the incidence and mortality rate of pancreatic cancer continue to be on the rise [4]. Therefore, pancreatic cancer is one of the most significant cancers affecting the health of the people in China and worldwide, which has caused serious economic burden to the families of the patients $[5,6]$. Therefore, new methods are needed to overcome the invasion, metastasis, and drug resistance of pancreatic cancers.

Several studies have investigated the possible molecular mechanisms of pancreatic cancer initiation and development to identify novel therapeutic targets for pancreatic cancer $[7,8]$. Notably, noncoding RNA transcripts such as microRNAs (miRNAs) and long noncoding RNAs (IncRNAs) have been reported to play significant roles in the molecular mechanism of cancers $[9,10]$. According to the competing endogenous RNA (ceRNA) hypothesis, different RNA transcripts participate in pathological processes mainly through competing for the binding sites of shared miRNAs. Fu et al. [11] analyzed the relationship between the expression of the LncRNA, HOTTIP, and overall or disease-free survival of 90 patients with pancreatic cancer following radical resection. They found that HOTTIP modulates cancer stem cell properties in human pancreatic cancer by regulating HOXA9. Cheng et al. [12] investigated the functional effects and the possible mechanisms of the small nucleolar RNA host gene 7 (SNHG7) in pancreatic cancer, and found that SNHG7 was overexpressed in pancreatic cancer tissues, and knockdown of

Page 2/38 
SNHG7 suppressed pancreatic cancer cell proliferation, migration, and invasion through the miR-342-3p/ID4 axis.

Small nucleolar noncoding RNAs (snoRNAs) are generally considered to be housekeeping molecules of ribosome function. In addition, subsets of snoRNAs were shown to be dysregulated in some cancers, including SNORA74B, SNORA21, U50, SNORD47, and SNORD62 [13, 14]. Alexey et al. [15] investigated the expression of six miRNAs isolated from formalin-fixed paraffin-embedded samples of pancreatic adenocarcinomas, and found that the snoRNA, U91, was a new internal control for accurate microRNA quantification in pancreatic cancer.

In this study, we screened differentially expressed snoRNAs (DEsnoRNAs) and trend-related snoRNAs based on the cancer genome atlas (TCGA) dataset for pancreatic cancer, and then performed methylation correlation analysis, survival analysis, and extraction of snoRNA host genes for Gene ontology (GO) functional and the Kyoto Encyclopedia of Genes and Genomes (KEGG) pathway enrichment analyses. Then, the DESNHGs and trend-related SNHGs were screened based on the TCGA dataset for pancreatic cancer, and a ceRNA network was constructed for pathway and functional enrichment analysis. A flow chart depicting the schematic of this study is shown in Fig. 1. This study aims to provide better understanding and promising therapeutic targets for pancreatic cancer.

\section{Materials And Methods}

\section{SnORNA}

\section{Data preprocessing}

The TCGA dataset for pancreatic cancer and the corresponding comment files were downloaded from UCSC Xena [16]. First, the data was re-annotated to extract the expression data for snoRNAs. Then the downloaded data was transformed to get the count values, and the count values were sorted to obtain the expression matrix of the data. Lastly, details about the pancreatic cancer of the sample and the stage grouping were obtained. The grouping of pancreatic cancer included tumor and normal time-series analysis of the stage.

\section{Identification of differentially expressed snoRNAs}

The differential analysis compared the tumor group with the normal. First, based on the read count data of the snoRNAs, the TMM (trimmed mean of $m$ values) normalization provided by the $\mathrm{R}$ software package edge $[17,18]$ was used to preprocess the read counts. Then the quasi-likelihood (QL) F-test in the edgeR package was applied for screening the differentially expressed snoRNAs (DEsnoRNAs), with the screening criteria set as $p<0.05,|\log F C|>1$ (foldchange > 1.2). Based on the DEsnoRNA values obtained from the screening, a heat map was drawn to observe the clustering of samples, and a volcano plot was drawn to show the difference.

\section{Identification of trend-related snoRNAs}

Based on the classification of the tumor stage diagnosis, including stage I, stage II, stage III, and stage IV, the data were analyzed using the STEM [19] software (version 1.3.11, http://www.cs.cmu.edu/ jernst/stem/) for trend analysis. The data were processed with 'No normalization/add 0 ' and screened with $p<0.05$.

\section{Identification of host genes of DEsnoRNAs and trend-related snoRNAs}

The host genes of DEsnoRNAs and trend-related snoRNAs in the data were extracted using the snoopy [20] tool (http://snoopy.med.miyazaki-u.ac.jp/). 


\section{GO functional and pathway enrichment analysis}

According to the host genes of the snoRNAs, the clusterprofiler tool [21] was used to perform GO functional and KEGG pathway analysis. The GO functional analysis included biological processes (BP), cellular components (CC), and molecular functions (MF).

\section{Survival analysis}

Survival analysis was conducted on the DEsnoRNA and trend-related snoRNA data. $p<0.05$ was considered to be an association with survival and prognosis. During the analysis, samples with survival information were screened and those with survival time longer than one month were selected.

\section{Methylation correlation analysis}

The methylation correlation analysis was conducted using the DEsnoRNAs, trend-related snoRNAs, and the data of TCGA methylation with corresponding pancreatic cancer. Then the methylation correlation results were obtained using the SNORic tool [22] ( http://bioinfo.life.hust.edu.cn/SNORic/) .

\section{SNHG}

\section{Data preprocessing}

The TCGA dataset for pancreatic cancer and the corresponding comment files were downloaded from UCSC Xena [16]. First, the data was re-annotated to extract the expression data for small nucleolar RNA host gene (SNHG), and the mRNA expression data corresponding to each dataset was extracted. The downloaded data were transformed to get the count values, and the count values were sorted to obtain the expression matrix of the data. Finally, details about the pancreatic cancer of the sample and the stage grouping were obtained. The grouping of pancreatic cancer included tumor and normal time-series analysis of stage analysis.

\section{Identification of differentially expressed SNHGs}

Differential analysis was used to compare the tumor and normal groups. First, according to the read count data, the TMM (trimmed mean of $\mathrm{m}$ values) normalization provided by the $\mathrm{R}$ software package edge $[17,18]$ was used to preprocess the read count. Then, the quasi-likelihood (QL) F-test in the edgeR package was applied for screening the DESNHGs, and the following screening criteria were used $p<0.05,|\log F C|>0.585$ (foldchange $>1.5$ ).

\section{Identification of trend-related SNHGs}

Based on the classification of tumor stage diagnosis, including stage I, stage II, stage III and stage IV, the data were processed using the STEM [19] software (version 1.3.11,

http://www.cs.cmu.edu/ jernst/stem/ ) for trend analysis. First, the data were processed with 'No normalization/add 0 ' and screened with $\mathrm{p}<0.05$ parameter. Then the data were processed according to the parameter 'normalize data' and screened with $p<0.1$.

\section{Correlation analysis}

Based on the results of the differential and trend analyses, the SNHGs related to the pancreatic cancer trend were selected and combined with the mRNA expression value of corresponding datasets. LncRNA and its corresponding 
mRNA in each group of data were analyzed to obtain the IncRNA-mRNA interaction pairs. The screening threshold was set at $\mathrm{p}<0.05$.

\section{Prediction of IncRNA-miRNA interaction pairs}

The Miranda [23] software was used to extract the IncRNA sequence in the IncRNA-mRNA interaction pairs, and to predict the target miRNA. Then the results were screened with the screening parameters -sc 140, -en -20, and the IncRNA-miRNA interaction pairs were obtained.

\section{Prediction of mRNA-miRNA interaction pairs}

The Miranda [23] software was used to extract the mRNA sequence in the IncRNA-mRNA interaction pairs, and to predict the target miRNA. Then the results were screened with the screening parameters -sc 140, -en -20, and the mRNA-miRNA interaction pairs were obtained.

\section{Construction of ceRNA network}

The interaction pairs of IncRNA-mRNA, IncRNA-miRNA, and mRNA-miRNA were integrated to construct the ceRNA network and to obtain the ceRNA interaction pairs.

\section{GO functional and pathway enrichment analysis in ceRNA network}

The genes in the ceRNA network were used to perform KEGG pathway annotation using the clusterprofiler tool [21]. Then the pathways that may be affected by the SNHGs were obtained, and the mechanism of its effect on pancreatic cancer was analyzed.

\section{Statistical analysis}

SPSS 22.0 statistical software was used for statistical processing. Continuous variables are expressed as mean \pm standard deviation (mean \pm SD). The enumeration data consistent with normal distribution between two groups were compared using the $t$ test, and the comparison among groups of enumeration data were tested using chi-square test. Statistical difference with $p<0.05$ was considered significant.

\section{Results}

\section{Clinical information of pancreatic cancer data samples}

As shown in Table 1, the sites of pancreatic cancer included the body of pancreas, head of pancreas, overlapping lesion of pancreas, pancreas, NOS, and tail of pancreas. There was a significant difference between stage I, II, III, and IV $(p<0.05)$. The type and age of pancreatic cancer between stage I, II, III, and IV showed no significant differences. 
Table 1

Clinical information of pancreatic cancer data sample

\begin{tabular}{|c|c|c|c|c|c|c|}
\hline Stage & & I & II & III & IV & $\begin{array}{l}P \\
\text { value }\end{array}$ \\
\hline Age & & $\begin{array}{l}65.57 \pm \\
12.13\end{array}$ & $\begin{array}{l}64.68 \pm \\
10.97\end{array}$ & $\begin{array}{l}65.67 \pm \\
15.28\end{array}$ & $\begin{array}{l}65.80 \pm \\
4.92\end{array}$ & 0.27 \\
\hline \multirow[t]{2}{*}{ Gender } & male & 10 & 83 & 1 & 2 & \\
\hline & female & 11 & 65 & 2 & 3 & \\
\hline \multirow[t]{5}{*}{ Site } & Body of pancreas & 4 & 9 & 0 & 1 & 0.005 \\
\hline & Head of pancreas & 12 & 112 & 3 & 1 & \\
\hline & Overlapping lesion of pancreas & 0 & 2 & 0 & 0 & \\
\hline & Pancreas, NOS & 1 & 16 & 0 & 0 & \\
\hline & Tail of pancreas & 4 & 9 & 0 & 3 & \\
\hline \multirow[t]{4}{*}{ Type } & Adenomas and Adenocarcinomas & 7 & 21 & 0 & 2 & 0.68 \\
\hline & $\begin{array}{l}\text { Cystic, Mucinous and Serous } \\
\text { Neoplasms }\end{array}$ & 1 & 4 & 0 & 0 & \\
\hline & Ductal and Lobular Neoplasms & 13 & 122 & 3 & 3 & \\
\hline & Epithelial Neoplasms, NOS & 0 & 1 & 0 & 0 & \\
\hline
\end{tabular}

\section{Snorna}

\section{Identification of differentially expressed snoRNAs and trend- related snoRNAs}

A total of eight DEsnoRNAs were identified using the method described above with $p<0.05$ and $\| \log F C \mid>1$ (foldchange $>1.2$ ) and consisted of two upregulated and six downregulated snoRNAs. The heatmap and volcano plot of the DEsnoRNAs are shown in Fig. 2. After the data were processed with 'No normalization/add 0' and screened with $p<0.05$, a total of 93 trend-related snoRNAs were obtained (Fig. 3, Table 2). The top 10 trend-related snoRNAs were selected for subsequent analysis. 
Table 2

Identification of trend-related snoRNAs

\begin{tabular}{|c|c|c|c|c|c|c|c|}
\hline Weight & snoRNA & Spot IDs & 0 & i & ii & iii & iv \\
\hline 1.00 & ACA59 & 1 & 0.00 & 8.94 & 9.05 & 10.59 & 10.87 \\
\hline 1.00 & SCARNA10 & 3 & 0.00 & 10.87 & 9.11 & 8.90 & 10.29 \\
\hline 1.00 & SCARNA12 & 4 & 0.00 & 13.41 & 13.09 & 13.51 & 13.09 \\
\hline 1.00 & SNORA11 & 5 & 0.00 & 10.75 & 10.54 & 10.80 & 10.45 \\
\hline 1.00 & SNORA12 & 6 & 0.00 & 11.50 & 10.84 & 11.08 & 11.12 \\
\hline 1.00 & SNORA13 & 7 & 0.00 & 12.63 & 12.76 & 12.59 & 12.78 \\
\hline 1.00 & SNORA14 & 8 & 0.00 & 4.79 & 6.20 & 12.26 & 4.82 \\
\hline 1.00 & SNORA14B & 9 & 0.00 & 10.39 & 10.23 & 12.90 & 10.07 \\
\hline 1.00 & SNORA16 & 11 & 0.00 & 10.42 & 9.40 & 11.98 & 8.96 \\
\hline 1.00 & SNORA16B & 12 & 0.00 & 8.97 & 10.06 & 7.78 & 13.58 \\
\hline 1.00 & SNORA19 & 13 & 0.00 & 3.04 & 4.60 & 6.70 & 9.25 \\
\hline 1.00 & SNORA2 & 14 & 0.00 & 12.29 & 12.12 & 12.32 & 12.32 \\
\hline 1.00 & SNORA20 & 15 & 0.00 & 3.71 & 4.75 & 6.08 & 3.83 \\
\hline 1.00 & SNORA22 & 16 & 0.00 & 9.67 & 9.87 & 7.35 & 10.71 \\
\hline 1.00 & SNORA23 & 17 & 0.00 & 8.27 & 8.42 & 12.46 & 7.88 \\
\hline 1.00 & SNORA26 & 18 & 0.00 & 9.81 & 9.54 & 9.68 & 9.60 \\
\hline 1.00 & SNORA3 & 21 & 0.00 & 5.60 & 5.52 & 9.09 & 7.76 \\
\hline 1.00 & SNORA31 & 22 & 0.00 & 8.65 & 8.85 & 8.76 & 9.06 \\
\hline 1.00 & SNORA33 & 23 & 0.00 & 12.04 & 11.91 & 12.10 & 11.89 \\
\hline 1.00 & SNORA34 & 24 & 0.00 & 11.18 & 10.02 & 12.46 & 10.05 \\
\hline 1.00 & SNORA38B & 25 & 0.00 & 6.33 & 6.46 & 8.27 & 7.64 \\
\hline 1.00 & SNORA40 & 27 & 0.00 & 8.25 & 8.21 & 8.42 & 8.34 \\
\hline 1.00 & SNORA42 & 28 & 0.00 & 8.69 & 8.28 & 6.97 & 9.73 \\
\hline 1.00 & SNORA44 & 29 & 0.00 & 4.98 & 7.44 & 7.20 & 11.57 \\
\hline 1.00 & SNORA45B & 30 & 0.00 & 12.18 & 12.33 & 8.86 & 13.55 \\
\hline 1.00 & SNORA46 & 31 & 0.00 & 11.23 & 10.70 & 11.73 & 11.93 \\
\hline 1.00 & SNORA47 & 32 & 0.00 & 7.73 & 7.03 & 4.70 & 9.57 \\
\hline 1.00 & SNORA48 & 33 & 0.00 & 4.65 & 5.01 & 5.30 & 4.48 \\
\hline 1.00 & SNORA51 & 34 & 0.00 & 6.67 & 8.42 & 9.26 & 9.25 \\
\hline 1.00 & SNORA53 & 35 & 0.00 & 12.56 & 12.85 & 13.51 & 13.32 \\
\hline
\end{tabular}




\begin{tabular}{|c|c|c|c|c|c|c|c|}
\hline Weight & snoRNA & Spot IDs & 0 & $\mathbf{i}$ & ii & iii & iv \\
\hline 1.00 & SNORA54 & 36 & 0.00 & 7.31 & 8.25 & 8.44 & 7.28 \\
\hline 1.00 & SNORA55 & 37 & 0.00 & 11.61 & 12.49 & 8.74 & 14.56 \\
\hline 1.00 & SNORA58 & 38 & 0.00 & 9.68 & 8.13 & 6.74 & 8.45 \\
\hline 1.00 & SNORA59A & 39 & 0.00 & 11.39 & 12.06 & 12.27 & 12.26 \\
\hline 1.00 & SNORA5A & 40 & 0.00 & 13.11 & 11.86 & 9.11 & 13.09 \\
\hline 1.00 & SNORA5C & 41 & 0.00 & 14.83 & 14.80 & 13.70 & 14.60 \\
\hline 1.00 & SNORA60 & 42 & 0.00 & 14.14 & 13.36 & 13.45 & 13.85 \\
\hline 1.00 & SNORA63 & 43 & 0.00 & 8.17 & 7.49 & 5.91 & 8.93 \\
\hline 1.00 & SNORA65 & 44 & 0.00 & 15.08 & 14.69 & 15.16 & 14.96 \\
\hline 1.00 & SNORA66 & 45 & 0.00 & 13.96 & 14.24 & 13.42 & 14.66 \\
\hline 1.00 & SNORA67 & 46 & 0.00 & 6.65 & 5.54 & 6.25 & 8.45 \\
\hline 1.00 & SNORA68 & 47 & 0.00 & 6.58 & 6.64 & 9.04 & 7.47 \\
\hline 1.00 & SNORA69 & 48 & 0.00 & 10.52 & 10.74 & 12.28 & 11.33 \\
\hline 1.00 & SNORA7 & 49 & 0.00 & 10.53 & 9.90 & 10.77 & 10.65 \\
\hline 1.00 & SNORA70F & 51 & 0.00 & 5.13 & 7.85 & 12.26 & 12.20 \\
\hline 1.00 & SNORA71A & 52 & 0.00 & 12.55 & 12.87 & 13.42 & 13.90 \\
\hline 1.00 & SNORA71B & 53 & 0.00 & 13.20 & 13.28 & 13.53 & 14.19 \\
\hline 1.00 & SNORA71C & 54 & 0.00 & 11.40 & 12.94 & 8.97 & 13.75 \\
\hline 1.00 & SNORA72 & 55 & 0.00 & 7.19 & 5.76 & 5.70 & 6.73 \\
\hline 1.00 & SNORA73B & 56 & 0.00 & 15.03 & 15.15 & 15.46 & 15.45 \\
\hline 1.00 & SNORA74 & 57 & 0.00 & 6.29 & 5.86 & 5.85 & 5.96 \\
\hline 1.00 & SNORA75 & 59 & 0.00 & 9.28 & 9.78 & 9.62 & 9.57 \\
\hline 1.00 & SNORA77 & 60 & 0.00 & 7.59 & 9.65 & 10.57 & 9.76 \\
\hline 1.00 & SNORA81 & 64 & 0.00 & 10.37 & 10.78 & 10.93 & 10.75 \\
\hline 1.00 & SNORA9 & 65 & 0.00 & 8.61 & 9.07 & 10.02 & 9.91 \\
\hline 1.00 & SNORD100 & 66 & 0.00 & 14.40 & 14.49 & 14.58 & 14.86 \\
\hline 1.00 & SNORD101 & 67 & 0.00 & 12.34 & 12.31 & 12.98 & 13.69 \\
\hline 1.00 & SNORD104 & 68 & 0.00 & 16.64 & 16.58 & 16.44 & 16.18 \\
\hline 1.00 & SNORD11 & 69 & 0.00 & 6.57 & 6.37 & 3.39 & 10.26 \\
\hline 1.00 & SNORD116-24 & 70 & 0.00 & 8.69 & 9.22 & 9.05 & 13.32 \\
\hline 1.00 & SNORD116-4 & 71 & 0.00 & 8.34 & 9.42 & 8.61 & 11.80 \\
\hline
\end{tabular}




\begin{tabular}{|c|c|c|c|c|c|c|c|}
\hline Weight & snoRNA & Spot IDs & 0 & $\mathbf{i}$ & ii & iii & iv \\
\hline 1.00 & SNORD117 & 72 & 0.00 & 8.14 & 10.08 & 8.64 & 13.22 \\
\hline 1.00 & SNORD12B & 75 & 0.00 & 10.69 & 12.83 & 9.30 & 14.29 \\
\hline 1.00 & SNORD12C & 76 & 0.00 & 6.49 & 7.27 & 8.08 & 9.58 \\
\hline 1.00 & SNORD14A & 77 & 0.00 & 15.81 & 15.51 & 15.53 & 14.92 \\
\hline 1.00 & SNORD14E & 78 & 0.00 & 13.59 & 14.07 & 15.11 & 13.54 \\
\hline 1.00 & SNORD15B & 80 & 0.00 & 11.15 & 12.10 & 12.90 & 13.06 \\
\hline 1.00 & SNORD17 & 81 & 0.00 & 13.88 & 14.27 & 14.58 & 14.47 \\
\hline 1.00 & SNORD19 & 82 & 0.00 & 12.39 & 12.17 & 11.55 & 12.85 \\
\hline 1.00 & SNORD1B & 83 & 0.00 & 5.66 & 6.67 & 7.67 & 6.98 \\
\hline 1.00 & SNORD46 & 85 & 0.00 & 10.46 & 10.74 & 10.30 & 11.47 \\
\hline 1.00 & SNORD51 & 86 & 0.00 & 6.73 & 8.46 & 7.78 & 11.04 \\
\hline 1.00 & SNORD53_SNORD92 & 87 & 0.00 & 6.16 & 6.56 & 3.85 & 9.48 \\
\hline 1.00 & SNORD56 & 88 & 0.00 & 8.90 & 8.99 & 9.60 & 9.77 \\
\hline 1.00 & SNORD59A & 89 & 0.00 & 8.54 & 9.87 & 8.27 & 12.78 \\
\hline 1.00 & SNORD 6 & 90 & 0.00 & 14.68 & 14.81 & 14.32 & 14.58 \\
\hline 1.00 & SNORD60 & 91 & 0.00 & 11.76 & 11.36 & 10.55 & 11.73 \\
\hline 1.00 & SNORD62B & 92 & 0.00 & 13.01 & 12.67 & 13.32 & 13.73 \\
\hline 1.00 & SNORD63 & 93 & 0.00 & 10.89 & 11.22 & 11.23 & 11.92 \\
\hline 1.00 & SNORD67 & 94 & 0.00 & 4.55 & 6.08 & 7.09 & 4.54 \\
\hline 1.00 & SNORD69 & 95 & 0.00 & 12.30 & 12.42 & 12.26 & 13.54 \\
\hline 1.00 & SNORD72 & 97 & 0.00 & 6.93 & 9.79 & 12.59 & 10.43 \\
\hline 1.00 & SNORD83A & 99 & 0.00 & 11.45 & 12.03 & 12.98 & 10.20 \\
\hline 1.00 & SNORD88A & 101 & 0.00 & 5.11 & 6.10 & 4.15 & 12.26 \\
\hline 1.00 & SNORD89 & 102 & 0.00 & 13.28 & 14.16 & 14.52 & 14.09 \\
\hline 1.00 & SNORD9 & 103 & 0.00 & 8.71 & 7.72 & 8.30 & 9.55 \\
\hline 1.00 & SNORD91A & 104 & 0.00 & 7.16 & 6.47 & 7.09 & 10.49 \\
\hline 1.00 & SNORD94 & 106 & 0.00 & 14.70 & 14.73 & 15.38 & 15.26 \\
\hline 1.00 & SNORD99 & 107 & 0.00 & 14.86 & 15.30 & 14.47 & 15.49 \\
\hline 1.00 & SNOU109 & 108 & 0.00 & 10.27 & 9.97 & 10.31 & 10.29 \\
\hline 1.00 & SNOU13 & 109 & 0.00 & 8.31 & 8.38 & 8.27 & 8.81 \\
\hline 1.00 & SNOZ196 & 110 & 0.00 & 11.66 & 12.09 & 8.52 & 12.84 \\
\hline
\end{tabular}




\section{Weight snoRNA Spot IDs $0 \quad$ i $\quad$ ii $\quad$ iii iv

$\begin{array}{llllllll}1.00 & \text { U3 } & 112 & 0.00 & 9.00 & 8.73 & 8.68 & 8.88\end{array}$ \\ Identification of host genes of DEsnoRNAs and trend-related snoRNAs}

In total, four host genes of the DEsnoRNAs in the data were obtained, including NCAPD2, C1orf79, NAP1L4, and $S N X 5$. A total of six host genes of the trend-related snoRNAs in data were identified, including NCAPD2, PHB2, MAGED2, CWF19L1, C5orf26, and C1orf79 (Table 3).

Table 3

Host genes of DEsnoRNAs and trend-related snoRNAs

\begin{tabular}{|c|c|c|c|c|c|c|c|}
\hline $\begin{array}{l}\text { snoRNA } \\
\text { name }\end{array}$ & Species & $\begin{array}{l}\text { snoRNA } \\
\text { name }\end{array}$ & Box & $\begin{array}{l}\text { Target } \\
\text { RNA }\end{array}$ & Organization & Locus & name2 \\
\hline \multicolumn{8}{|l|}{ DEsnoRNAs } \\
\hline SCARNA10 & Homo_sapiens & $\begin{array}{l}\text { SCARNA10 } \\
\text { (U85) }\end{array}$ & "H/ACA,C/D" & $\begin{array}{l}\text { U5 } \\
\text { snRNA }\end{array}$ & Intronic & NCAPD2 & U85 \\
\hline SNORA44 & Homo_sapiens & $\begin{array}{l}\text { SNORA44 } \\
\text { (ACA44) }\end{array}$ & $\mathrm{H} / \mathrm{ACA}$ & $\begin{array}{l}\text { 18S } \\
\text { rRNA }\end{array}$ & Intronic & C1orf79 & ACA44 \\
\hline SNORA54 & Homo_sapiens & $\begin{array}{l}\text { SNORA54 } \\
\text { (ACA54) }\end{array}$ & $\mathrm{H} / \mathrm{ACA}$ & $\begin{array}{l}28 S \\
\text { rRNA }\end{array}$ & Intronic & NAP1L4 & ACA54 \\
\hline SNORD17 & Homo_sapiens & $\begin{array}{l}\text { SNORD17 } \\
\text { (HBI-43) }\end{array}$ & $C / D$ & $\begin{array}{l}28 S \\
\text { rRNA }\end{array}$ & Intronic & SNX5 & $\mathrm{HBI}-43$ \\
\hline \multicolumn{8}{|c|}{ trend-related snoRNAs } \\
\hline SCARNA10 & Homo sapiens & $\begin{array}{l}\text { SCARNA10 } \\
\text { (U85) }\end{array}$ & "H/ACA,C/D" & $\begin{array}{l}\text { U5 } \\
\text { snRNA }\end{array}$ & Intronic & NCAPD2 & U85 \\
\hline SCARNA12 & Homo sapiens & $\begin{array}{l}\text { SCARNA12 } \\
\text { (U89) }\end{array}$ & $\mathrm{H} / \mathrm{ACA}$ & $\begin{array}{l}\text { U5 } \\
\text { snRNA }\end{array}$ & Intronic & PHB2 & U89 \\
\hline SNORA11 & Homo sapiens & $\begin{array}{l}\text { SNORA11 } \\
\text { (U107) }\end{array}$ & $\mathrm{H} / \mathrm{ACA}$ & Unknown & Intronic & MAGED2 & U107 \\
\hline SNORA12 & Homo sapiens & $\begin{array}{l}\text { SNORA12 } \\
\text { (U108) }\end{array}$ & $\mathrm{H} / \mathrm{ACA}$ & Unknown & Intronic & CWF19L1 & U108 \\
\hline SNORA13 & Homo sapiens & $\begin{array}{l}\text { SNORA13 } \\
\text { (ACA13) }\end{array}$ & $\mathrm{H} / \mathrm{ACA}$ & $\begin{array}{l}\text { 18S } \\
\text { rRNA }\end{array}$ & intronic & C5orf26 & ACA13 \\
\hline SNORA16 & Homo sapiens & $\begin{array}{l}\text { SNORA16 } \\
\text { (ACA16) }\end{array}$ & $\mathrm{H} / \mathrm{ACA}$ & $\begin{array}{l}28 S \\
\text { rRNA }\end{array}$ & Intronic & C1orf79 & ACA16 \\
\hline
\end{tabular}

\section{Functional analysis of host genes of DEsnoRNAs and trend-related snoRNAs}

The GO enrichment analysis of the host genes of DEsnoRNAs revealed that the significantly enriched GO-BP terms included mitotic chromosome condensation, chromosome condensation, and mitotic sister chromatid segregation. The condensin complex, DNA packaging complex, and condensed chromosome were three markedly enriched GO-CC terms. Furthermore, we also found that these genes played essential roles in histone binding, nucleosome binding, 
and actin filament binding according to the GO-MF analysis. The KEGG pathway analysis suggested that these genes were responsible for endocytosis pathway. The GO enrichment analysis of the host genes of trend-related snoRNAs revealed that the significantly enriched GO-BP terms included mitotic chromosome condensation, chromosome condensation, and mitotic sister chromatid segregation. The condensin complex, DNA packaging complex, and condensed chromosome were three markedly enriched GO-CC terms. Furthermore, we also found that these genes played essential roles in histone binding, nucleosome binding, and estrogen receptor binding according to the GO-MF analysis. The KEGG pathway analysis revealed that these genes were responsible for the endocytosis pathway (Fig. 4 and Supplementary file 1).

\section{Survival analysis of DEsnoRNAs and trend-related snoRNAs}

snoRNA survival analysis was performed based on the DEsnoRNAs and trend-related snoRNAs. As shown in the Fig. 5B, SNORA38B, a DEsnoRNA with $p=0.0092$, was found to be associated with survival and prognosis. The survival analysis of the top 10 trend-related snoRNA are shown in Fig. 5.

\section{Methylation correlation analysis of DEsnoRNAs and trend-related snoRNAs}

Methylation correlation analysis was performed based on the DEsnoRNAs and trend-related snoRNAs. As illustrated in Fig. 6 and Table 4, SNORD17, a DEsnoRNA with the $p=0.0412$ and correlation coefficient $=-0.2077$, was found to be negatively correlated with methylation. SNORA11, a trend-related snoRNA, was also found to be negatively correlated with methylation ( $p=0.0336$ and correlation coefficient $=-0.2159$ ).

Table 4

Methylation correlation analysis of DEsnoRNAs and trend-related snoRNAs

\begin{tabular}{|llll|}
\hline snoRNA & Chip ID & Coefficient & P-value \\
\hline ACA54; ENSG00000207008; SNORA54 & cg26350143 & 0.0831 & 0.4187 \\
\hline ACA54; ENSG00000207008; SNORA54 & cg03241897 & -0.0234 & 0.8201 \\
\hline HBI-43; ENSG00000212232; SNORD17 & cg07577824 & -0.082 & 0.4245 \\
\hline HBI-43; ENSG00000212232; SNORD17 & $\operatorname{cg} 17507897$ & -0.0823 & 0.4228 \\
\hline HBI-43; ENSG00000212232; SNORD17 & $\operatorname{cg} 02912291$ & -0.1451 & 0.1561 \\
\hline HBI-43; ENSG00000212232; SNORD17 & $\operatorname{cg} 22145445$ & -0.2077 & 0.0412 \\
\hline U107; ENSG00000221716; SNORA11 & $\operatorname{cg} 23090195$ & -0.1127 & 0.2718 \\
\hline U107; ENSG00000221716; SNORA11 & $\operatorname{cg} 18528798$ & -0.1455 & 0.1549 \\
\hline U107; ENSG00000221716; SNORA11 & $\operatorname{cg} 14338160$ & -0.1818 & 0.0747 \\
\hline U107; ENSG00000221716; SNORA11 & $\operatorname{cg} 22072691$ & -0.2159 & 0.0336 \\
\hline
\end{tabular}

SNHG

Identification of differentially expressed SNHGs and trend-related SNHGs 
Based on $p<0.05,|\log F C|>0.585$ (foldchange $>1.5$ ), no DESNHG was identified. After the data were processed with 'No normalization/add 0 ' and screened with $p<0.05$, there were no significant trend-related SNHGs. Then the data were processed based on the parameter 'normalize data' and screened with $p<0.1$, and two SNHGs correlated with trends, including SNHG23 and SNHG24 (Fig. 7, Table 5). The expression values of these two SNHGs were selected for subsequent analysis.

Table 5

Identification of trend-related SNHGs

\begin{tabular}{|c|c|c|c|c|c|c|c|c|}
\hline Weight & stage & Spot IDs & 0 & stage i & stage ii & stage iii & stage iv & \\
\hline \multicolumn{9}{|c|}{ No normalize/add0 } \\
\hline 1.00 & SNHG16 & 1 & 0.00 & 15.05 & 15.33 & 15.70 & 15.73 & \\
\hline 1.00 & SNHG11 & 2 & 0.00 & 14.47 & 14.46 & 14.36 & 14.28 & \\
\hline 1.00 & SNHG17 & 3 & 0.00 & 15.40 & 15.54 & 15.48 & 15.90 & \\
\hline 1.00 & SNHG12 & 4 & 0.00 & 14.56 & 15.28 & 14.77 & 15.27 & \\
\hline 1.00 & SNHG5 & 5 & 0.00 & 17.18 & 17.58 & 18.23 & 17.92 & \\
\hline 1.00 & SNHG14 & 6 & 0.00 & 16.03 & 15.65 & 15.71 & 15.90 & \\
\hline 1.00 & SNHG23 & 7 & 0.00 & 8.53 & 7.98 & 9.65 & 8.44 & \\
\hline 1.00 & SNHG15 & 8 & 0.00 & 15.09 & 15.28 & 15.32 & 15.42 & \\
\hline 1.00 & SNHG7 & 9 & 0.00 & 16.42 & 16.12 & 16.34 & 16.14 & \\
\hline 1.00 & SNHG20 & 10 & 0.00 & 13.75 & 13.57 & 13.31 & 13.71 & \\
\hline 1.00 & SNHG3 & 11 & 0.00 & 15.20 & 15.43 & 15.09 & 15.33 & \\
\hline 1.00 & SNHG6 & 12 & 0.00 & 16.95 & 16.90 & 17.16 & 16.68 & \\
\hline 1.00 & SNHG10 & 13 & 0.00 & 13.23 & 13.03 & 12.81 & 13.02 & \\
\hline 1.00 & SNHG18 & 14 & 0.00 & 13.63 & 14.36 & 14.57 & 14.41 & \\
\hline 1.00 & SNHG21 & 15 & 0.00 & 11.26 & 11.10 & 11.25 & 10.96 & \\
\hline 1.00 & SNHG9 & 16 & 0.00 & 13.27 & 12.74 & 12.81 & 12.65 & \\
\hline 1.00 & SNHG1 & 17 & 0.00 & 15.89 & 16.25 & 15.99 & 16.34 & \\
\hline 1.00 & SNHG19 & 18 & 0.00 & 14.55 & 13.90 & 13.83 & 13.89 & \\
\hline 1.00 & SNHG22 & 19 & 0.00 & 10.71 & 10.84 & 10.58 & 10.44 & \\
\hline 1.00 & SNHG8 & 20 & 0.00 & 16.90 & 16.48 & 16.80 & 16.57 & \\
\hline 1.00 & SNHG24 & 21 & 0.00 & 7.59 & 7.22 & 7.92 & 6.92 & \\
\hline 1.00 & SNHG4 & 22 & 0.00 & 10.70 & 11.84 & 10.96 & 11.93 & \\
\hline \multicolumn{9}{|c|}{ Normalize } \\
\hline 1.00 & SNHG23 & 7 & & & 0.00 & -0.55 & 1.13 & -0.09 \\
\hline 1.00 & SNHG24 & 21 & & & 0.00 & -0.37 & 0.33 & -0.67 \\
\hline
\end{tabular}




\section{Identification of IncRNA-mRNA interaction pairs}

Based on the SNHGs related to the pancreatic cancer trend, the data of the IncRNA and the corresponding mRNAs in each group were analyzed with the screening threshold $p<0.05$. The result revealed two IncRNA-mRNA interaction pairs, including SNHG24-FAM25A and SNHG24-KISS1.

\section{Prediction of IncRNA-miRNA and mRNA-miRNA interaction pairs}

With the screening parameters of -sc 140 and -en -20, a total of 127 IncRNA-miRNA interaction pairs were obtained. The IncRNA-miRNA interaction pairs are shown in Table 6 . With the screening parameters of -sc 140 and -en -20, a total 99 mRNA-miRNA interaction pairs were identified. The mRNA-miRNA interaction pairs are shown in Table 7. 
Table 6

Prediction of IncRNA-miRNA interaction pairs

\begin{tabular}{|c|c|c|c|c|c|c|c|c|c|}
\hline Seq1 & Seq2 & $\begin{array}{l}\text { Tot } \\
\text { Score }\end{array}$ & $\begin{array}{l}\text { Tot } \\
\text { Energy }\end{array}$ & $\begin{array}{l}\text { Max } \\
\text { Score }\end{array}$ & $\begin{array}{l}\text { Max } \\
\text { Energy }\end{array}$ & Strand & Len1 & Len2 & Positions \\
\hline hsa-miR-17-5p & SNHG24 & 169 & -24.75 & 169 & -24.75 & 19 & 23 & 1634 & 879 \\
\hline $\begin{array}{l}\text { hsa-miR-19b- } \\
1-5 p\end{array}$ & SNHG24 & 149 & -26.29 & 149 & -26.29 & 25 & 23 & 1634 & 775 \\
\hline $\begin{array}{l}\text { hsa-miR-20a- } \\
5 p\end{array}$ & SNHG24 & 165 & -21.46 & 165 & -21.46 & 28 & 23 & 1634 & 879 \\
\hline $\begin{array}{l}\text { hsa-miR-26a- } \\
1-3 p\end{array}$ & SNHG24 & 159 & -20.94 & 159 & -20.94 & 42 & 22 & 1634 & 447 \\
\hline hsa-miR-93-5p & SNHG24 & 169 & -25.95 & 169 & -25.95 & 62 & 23 & 1634 & 879 \\
\hline $\begin{array}{l}\text { hsa-miR-103a- } \\
3 p\end{array}$ & SNHG24 & 140 & -20.88 & 140 & -20.88 & 80 & 23 & 1634 & 662 \\
\hline $\begin{array}{l}\text { hsa-miR-106a- } \\
5 p\end{array}$ & SNHG24 & 169 & -20.51 & 169 & -20.51 & 84 & 23 & 1634 & 879 \\
\hline hsa-miR-107 & SNHG24 & 140 & -20.88 & 140 & -20.88 & 86 & 23 & 1634 & 662 \\
\hline $\begin{array}{l}\text { hsa-miR-23b- } \\
5 p\end{array}$ & SNHG24 & 143 & -21.29 & 143 & -21.29 & 177 & 22 & 1634 & 1441 \\
\hline $\begin{array}{l}\text { hsa-miR-132- } \\
3 p\end{array}$ & SNHG24 & 152 & -23.84 & 152 & -23.84 & 194 & 22 & 1634 & 4 \\
\hline $\begin{array}{l}\text { hsa-miR-140- } \\
3 p\end{array}$ & SNHG24 & 153 & -22.18 & 153 & -22.18 & 205 & 21 & 1634 & 246 \\
\hline $\begin{array}{l}\text { hsa-miR-152- } \\
3 p\end{array}$ & SNHG24 & 145 & -20.88 & 145 & -20.88 & 217 & 21 & 1634 & 129 \\
\hline $\begin{array}{l}\text { hsa-miR-302a- } \\
3 p\end{array}$ & SNHG24 & 147 & -20.34 & 147 & -20.34 & 278 & 23 & 1634 & 541 \\
\hline $\begin{array}{l}\text { hsa-miR-99b- } \\
3 p\end{array}$ & SNHG24 & 150 & -22.74 & 150 & -22.74 & 290 & 22 & 1634 & 490 \\
\hline $\begin{array}{l}\text { hsa-miR-302b- } \\
3 p\end{array}$ & SNHG24 & 151 & -20.92 & 151 & -20.92 & 309 & 23 & 1634 & 541 \\
\hline $\begin{array}{l}\text { hsa-miR-378a- } \\
5 p\end{array}$ & SNHG24 & 140 & -20.4 & 140 & -20.4 & 336 & 22 & 1634 & 264 \\
\hline $\begin{array}{l}\text { hsa-miR-380- } \\
5 p\end{array}$ & SNHG24 & 149 & -21.95 & 149 & -21.95 & 340 & 22 & 1634 & 1140 \\
\hline $\begin{array}{l}\text { hsa-miR-135b- } \\
3 p\end{array}$ & SNHG24 & 140 & -25.32 & 140 & -25.32 & 364 & 22 & 1634 & 81 \\
\hline $\begin{array}{l}\text { hsa-miR-20b- } \\
5 p\end{array}$ & SNHG24 & 165 & -24.08 & 165 & -24.08 & 394 & 23 & 1634 & 879 \\
\hline hsa-miR-484 & SNHG24 & 153 & -20.39 & 153 & -20.39 & 420 & 22 & 1634 & 943 \\
\hline $\begin{array}{l}\text { hsa-miR-485- } \\
5 p\end{array}$ & SNHG24 & 146 & -21.86 & 146 & -21.86 & 421 & 22 & 1634 & 324 \\
\hline
\end{tabular}




\begin{tabular}{|c|c|c|c|c|c|c|c|c|c|}
\hline Seq1 & Seq2 & $\begin{array}{l}\text { Tot } \\
\text { Score }\end{array}$ & $\begin{array}{l}\text { Tot } \\
\text { Energy }\end{array}$ & $\begin{array}{l}\text { Max } \\
\text { Score }\end{array}$ & $\begin{array}{l}\text { Max } \\
\text { Energy }\end{array}$ & Strand & Len1 & Len2 & Positions \\
\hline $\begin{array}{l}\text { hsa-miR-511- } \\
\text { 5p }\end{array}$ & SNHG24 & 141 & -27.13 & 141 & -27.13 & 435 & 21 & 1634 & 785 \\
\hline $\begin{array}{l}\text { hsa-miR-497- } \\
3 p\end{array}$ & SNHG24 & 150 & -20.13 & 150 & -20.13 & 454 & 22 & 1634 & 1081 \\
\hline $\begin{array}{l}\text { hsa-miR-519b- } \\
3 p\end{array}$ & SNHG24 & 142 & -20.1 & 142 & -20.1 & 476 & 22 & 1634 & 128 \\
\hline $\begin{array}{l}\text { hsa-miR-518a- } \\
5 p\end{array}$ & SNHG24 & 145 & -20.32 & 145 & -20.32 & 508 & 20 & 1634 & 798 \\
\hline hsa-miR-527 & SNHG24 & 145 & -20.32 & 145 & -20.32 & 518 & 20 & 1634 & 798 \\
\hline $\begin{array}{l}\text { hsa-miR-501- } \\
3 p\end{array}$ & SNHG24 & 155 & -20.7 & 155 & -20.7 & 527 & 22 & 1634 & 1030 \\
\hline $\begin{array}{l}\text { hsa-miR-450a- } \\
\text { 2-3p }\end{array}$ & SNHG24 & 143 & -22.98 & 143 & -22.98 & 530 & 22 & 1634 & 1317 \\
\hline $\begin{array}{l}\text { hsa-miR-585- } \\
5 \mathrm{p}\end{array}$ & SNHG24 & 164 & -28.23 & 164 & -28.23 & 607 & 22 & 1634 & 262 \\
\hline $\begin{array}{l}\text { hsa-miR-642a- } \\
5 p\end{array}$ & SNHG24 & 163 & -21.41 & 163 & -21.41 & 688 & 22 & 1634 & 1166 \\
\hline hsa-miR-657 & SNHG24 & 147 & -22.56 & 147 & -22.56 & 721 & 23 & 1634 & 770 \\
\hline $\begin{array}{l}\text { hsa-miR-758- } \\
3 p\end{array}$ & SNHG24 & 141 & -20.22 & 141 & -20.22 & 731 & 22 & 1634 & 1415 \\
\hline $\begin{array}{l}\text { hsa-miR-1301- } \\
3 p\end{array}$ & SNHG24 & 145 & -20.86 & 145 & -20.86 & 753 & 24 & 1634 & 84 \\
\hline hsa-miR-762 & SNHG24 & 147 & -30.34 & 147 & -30.34 & 767 & 22 & 1634 & 326 \\
\hline hsa-miR-759 & SNHG24 & 142 & -21.54 & 142 & -21.54 & 776 & 22 & 1634 & 422 \\
\hline $\begin{array}{l}\text { hsa-miR-942- } \\
\text { 5p }\end{array}$ & SNHG24 & 149 & -24.42 & 149 & -24.42 & 846 & 22 & 1634 & 1210 \\
\hline hsa-miR-1204 & SNHG24 & 146 & -22.06 & 146 & -22.06 & 884 & 21 & 1634 & 485 \\
\hline $\begin{array}{l}\text { hsa-miR-1304- } \\
5 p\end{array}$ & SNHG24 & 149 & -25.86 & 149 & -25.86 & 911 & 22 & 1634 & 39 \\
\hline $\begin{array}{l}\text { hsa-miR-1249- } \\
5 p\end{array}$ & SNHG24 & 140 & -27.44 & 140 & -27.44 & 923 & 24 & 1634 & 557 \\
\hline $\begin{array}{l}\text { hsa-miR-1266- } \\
5 p\end{array}$ & SNHG24 & 142 & -23.1 & 142 & -23.1 & 944 & 23 & 1634 & 1445 \\
\hline $\begin{array}{l}\text { hsa-miR-1910- } \\
3 p\end{array}$ & SNHG24 & 165 & -25.79 & 165 & -25.79 & 1004 & 20 & 1634 & 674 \\
\hline hsa-miR-2278 & SNHG24 & 142 & -21.48 & 142 & -21.48 & 1033 & 22 & 1634 & 1277 \\
\hline $\begin{array}{l}\text { hsa-miR-2681- } \\
3 p\end{array}$ & SNHG24 & 147 & -23.68 & 147 & -23.68 & 1035 & 22 & 1634 & 68 \\
\hline hsa-miR-3125 & SNHG24 & 156 & -20.05 & 156 & -20.05 & 1057 & 20 & 1634 & 1473 \\
\hline
\end{tabular}




\begin{tabular}{|c|c|c|c|c|c|c|c|c|c|}
\hline Seq1 & Seq2 & $\begin{array}{l}\text { Tot } \\
\text { Score }\end{array}$ & $\begin{array}{l}\text { Tot } \\
\text { Energy }\end{array}$ & $\begin{array}{l}\text { Max } \\
\text { Score }\end{array}$ & $\begin{array}{l}\text { Max } \\
\text { Energy }\end{array}$ & Strand & Len1 & Len2 & Positions \\
\hline $\begin{array}{l}\text { hsa-miR-3127- } \\
5 p\end{array}$ & SNHG24 & 291 & -54.17 & 147 & -32.51 & 1060 & 23 & 1634 & 148491 \\
\hline hsa-miR-378b & SNHG24 & 148 & -22.29 & 148 & -22.29 & 1070 & 19 & 1634 & 911 \\
\hline hsa-miR-548u & SNHG24 & 145 & -22.16 & 145 & -22.16 & 1087 & 23 & 1634 & 471 \\
\hline $\begin{array}{l}\text { hsa-miR- } \\
3150 a-5 p\end{array}$ & SNHG24 & 145 & -25.5 & 145 & -25.5 & 1098 & 22 & 1634 & 820 \\
\hline $\begin{array}{l}\text { hsa-miR-3151- } \\
5 p\end{array}$ & SNHG24 & 283 & -43.69 & 143 & -22.62 & 1100 & 21 & 1634 & $\begin{array}{l}917 \\
1005\end{array}$ \\
\hline hsa-miR-3178 & SNHG24 & 140 & -27.17 & 140 & -27.17 & 1141 & 17 & 1634 & 1005 \\
\hline $\begin{array}{l}\text { hsa-miR-3180- } \\
5 p\end{array}$ & SNHG24 & 151 & -22.38 & 151 & -22.38 & 1143 & 25 & 1634 & 1435 \\
\hline $\begin{array}{l}\text { hsa-miR-3187- } \\
3 p\end{array}$ & SNHG24 & 465 & -71.69 & 163 & -25.74 & 1157 & 20 & 1634 & $\begin{array}{l}483320 \\
236\end{array}$ \\
\hline $\begin{array}{l}\text { hsa-miR-3191- } \\
3 p\end{array}$ & SNHG24 & 144 & -28.29 & 144 & -28.29 & 1165 & 23 & 1634 & 1147 \\
\hline $\begin{array}{l}\text { hsa-miR-3194- } \\
3 p\end{array}$ & SNHG24 & 155 & -21.7 & 155 & -21.7 & 1170 & 22 & 1634 & 775 \\
\hline hsa-miR-4298 & SNHG24 & 141 & -20.93 & 141 & -20.93 & 1192 & 22 & 1634 & 1147 \\
\hline hsa-miR-4304 & SNHG24 & 146 & -24.11 & 146 & -24.11 & 1194 & 17 & 1634 & 666 \\
\hline $\begin{array}{l}\text { hsa-miR-3614- } \\
5 p\end{array}$ & SNHG24 & 141 & -22.32 & 141 & -22.32 & 1279 & 23 & 1634 & 910 \\
\hline $\begin{array}{l}\text { hsa-miR-3617- } \\
3 p\end{array}$ & SNHG24 & 164 & -21.7 & 164 & -21.7 & 1285 & 23 & 1634 & 798 \\
\hline $\begin{array}{l}\text { hsa-miR- } \\
3622 b-3 p\end{array}$ & SNHG24 & 152 & -23.87 & 152 & -23.87 & 1296 & 21 & 1634 & 459 \\
\hline $\begin{array}{l}\text { hsa-miR-3663- } \\
5 p\end{array}$ & SNHG24 & 292 & -42.98 & 150 & -22.86 & 1311 & 21 & 1634 & $\begin{array}{l}279 \\
1040\end{array}$ \\
\hline $\begin{array}{l}\text { hsa-miR-3667- } \\
3 p\end{array}$ & SNHG24 & 141 & -21.02 & 141 & -21.02 & 1318 & 22 & 1634 & 1024 \\
\hline $\begin{array}{l}\text { hsa-miR-3678- } \\
3 p\end{array}$ & SNHG24 & 147 & -21.26 & 147 & -21.26 & 1329 & 22 & 1634 & 1489 \\
\hline hsa-miR-3916 & SNHG24 & 140 & -22.49 & 140 & -22.49 & 1367 & 26 & 1634 & 670 \\
\hline $\begin{array}{l}\text { hsa-miR- } \\
\text { 3150b-5p }\end{array}$ & SNHG24 & 145 & -27.52 & 145 & -27.52 & 1371 & 22 & 1634 & 820 \\
\hline hsa-miR-3945 & SNHG24 & 143 & -21.37 & 143 & -21.37 & 1405 & 23 & 1634 & 1142 \\
\hline $\begin{array}{l}\text { hsa-miR- } \\
1268 b\end{array}$ & SNHG24 & 141 & -28.91 & 141 & -28.91 & 1415 & 20 & 1634 & 1000 \\
\hline hsa-miR-4441 & SNHG24 & 152 & -20.26 & 152 & -20.26 & 1449 & 17 & 1634 & 658 \\
\hline
\end{tabular}




\begin{tabular}{|c|c|c|c|c|c|c|c|c|c|}
\hline Seq1 & Seq2 & $\begin{array}{l}\text { Tot } \\
\text { Score }\end{array}$ & $\begin{array}{l}\text { Tot } \\
\text { Energy }\end{array}$ & $\begin{array}{l}\text { Max } \\
\text { Score }\end{array}$ & $\begin{array}{l}\text { Max } \\
\text { Energy }\end{array}$ & Strand & Len1 & Len2 & Positions \\
\hline $\begin{array}{l}\text { hsa-miR-4446- } \\
3 p\end{array}$ & SNHG24 & 141 & -21.87 & 141 & -21.87 & 1456 & 22 & 1634 & 330 \\
\hline hsa-miR-4462 & SNHG24 & 145 & -23.63 & 145 & -23.63 & 1475 & 23 & 1634 & 666 \\
\hline hsa-miR-4470 & SNHG24 & 297 & -43.74 & 151 & -22.13 & 1486 & 21 & 1634 & 541084 \\
\hline hsa-miR-4496 & SNHG24 & 162 & -27.52 & 162 & -27.52 & 1523 & 22 & 1634 & 1468 \\
\hline hsa-miR-4501 & SNHG24 & 296 & -41.82 & 151 & -21.78 & 1528 & 21 & 1634 & 742350 \\
\hline hsa-miR-4513 & SNHG24 & 142 & -23.92 & 142 & -23.92 & 1541 & 22 & 1634 & 1463 \\
\hline $\begin{array}{l}\text { hsa-miR-4520- } \\
5 p\end{array}$ & SNHG24 & 161 & -20.78 & 161 & -20.78 & 1548 & 20 & 1634 & 693 \\
\hline hsa-miR-4647 & SNHG24 & 144 & -21.07 & 144 & -21.07 & 1608 & 23 & 1634 & 929 \\
\hline $\begin{array}{l}\text { hsa-miR-4671- } \\
3 p\end{array}$ & SNHG24 & 142 & -22.58 & 142 & -22.58 & 1652 & 21 & 1634 & 128 \\
\hline $\begin{array}{l}\text { hsa-miR-1343- } \\
3 p\end{array}$ & SNHG24 & 147 & -22.41 & 147 & -22.41 & 1676 & 22 & 1634 & 41 \\
\hline hsa-miR-4688 & SNHG24 & 143 & -22.64 & 143 & -22.64 & 1677 & 22 & 1634 & 503 \\
\hline $\begin{array}{l}\text { hsa-miR-4691- } \\
5 p\end{array}$ & SNHG24 & 145 & -20.5 & 145 & -20.5 & 1681 & 23 & 1634 & 1 \\
\hline $\begin{array}{l}\text { hsa-miR-4695- } \\
5 p\end{array}$ & SNHG24 & 140 & -25.09 & 140 & -25.09 & 1688 & 22 & 1634 & 677 \\
\hline $\begin{array}{l}\text { hsa-miR-4717- } \\
3 p\end{array}$ & SNHG24 & 149 & -24.43 & 149 & -24.43 & 1730 & 21 & 1634 & 94 \\
\hline $\begin{array}{l}\text { hsa-miR-4732- } \\
5 p\end{array}$ & SNHG24 & 140 & -21.3 & 140 & -21.3 & 1756 & 23 & 1634 & 746 \\
\hline hsa-miR-4734 & SNHG24 & 143 & -25.03 & 143 & -25.03 & 1760 & 22 & 1634 & 772 \\
\hline hsa-miR-4739 & SNHG24 & 146 & -20.02 & 146 & -20.02 & 1769 & 25 & 1634 & 648 \\
\hline $\begin{array}{l}\text { hsa-miR-4756- } \\
5 p\end{array}$ & SNHG24 & 146 & -20.67 & 146 & -20.67 & 1802 & 23 & 1634 & 651 \\
\hline $\begin{array}{l}\text { hsa-miR-2467- } \\
5 p\end{array}$ & SNHG24 & 140 & -23.08 & 140 & -23.08 & 1855 & 23 & 1634 & 36 \\
\hline $\begin{array}{l}\text { hsa-miR-2467- } \\
3 p\end{array}$ & SNHG24 & 145 & -24.03 & 145 & -24.03 & 1856 & 22 & 1634 & 502 \\
\hline $\begin{array}{l}\text { hsa-miR-4793- } \\
5 p\end{array}$ & SNHG24 & 159 & -25.79 & 159 & -25.79 & 1867 & 24 & 1634 & 37 \\
\hline $\begin{array}{l}\text { hsa-miR-4802- } \\
3 p\end{array}$ & SNHG24 & 168 & -26.67 & 168 & -26.67 & 1884 & 23 & 1634 & 904 \\
\hline $\begin{array}{l}\text { hsa-miR-5088- } \\
5 p\end{array}$ & SNHG24 & 283 & -57.68 & 143 & -29.23 & 1918 & 24 & 1634 & 239488 \\
\hline
\end{tabular}




\begin{tabular}{|c|c|c|c|c|c|c|c|c|c|}
\hline Seq1 & Seq2 & $\begin{array}{l}\text { Tot } \\
\text { Score }\end{array}$ & $\begin{array}{l}\text { Tot } \\
\text { Energy }\end{array}$ & $\begin{array}{l}\text { Max } \\
\text { Score }\end{array}$ & $\begin{array}{l}\text { Max } \\
\text { Energy }\end{array}$ & Strand & Len1 & Len2 & Positions \\
\hline $\begin{array}{l}\text { hsa-miR-5189- } \\
3 p\end{array}$ & SNHG24 & 141 & -21.09 & 141 & -21.09 & 1932 & 21 & 1634 & 825 \\
\hline hsa-miR-5683 & SNHG24 & 154 & -20.92 & 154 & -20.92 & 1996 & 24 & 1634 & 255 \\
\hline hsa-miR-5691 & SNHG24 & 155 & -24.01 & 155 & -24.01 & 2006 & 22 & 1634 & 775 \\
\hline hsa-miR-6079 & SNHG24 & 162 & -20.2 & 162 & -20.2 & 2042 & 24 & 1634 & 222 \\
\hline $\begin{array}{l}\text { hsa-miR-6503- } \\
5 p\end{array}$ & SNHG24 & 470 & -66 & 173 & -25.22 & 2078 & 23 & 1634 & $\begin{array}{l}1036 \\
696862\end{array}$ \\
\hline $\begin{array}{l}\text { hsa-miR-6506- } \\
5 p\end{array}$ & SNHG24 & 145 & -20.49 & 145 & -20.49 & 2084 & 23 & 1634 & 1148 \\
\hline $\begin{array}{l}\text { hsa-miR-6730- } \\
5 p\end{array}$ & SNHG24 & 143 & -22.1 & 143 & -22.1 & 2130 & 23 & 1634 & 573 \\
\hline $\begin{array}{l}\text { hsa-miR-6739- } \\
3 p\end{array}$ & SNHG24 & 141 & -23.61 & 141 & -23.61 & 2149 & 21 & 1634 & 780 \\
\hline $\begin{array}{l}\text { hsa-miR-6743- } \\
5 p\end{array}$ & SNHG24 & 147 & -26.79 & 147 & -26.79 & 2156 & 22 & 1634 & 673 \\
\hline $\begin{array}{l}\text { hsa-miR-6753- } \\
5 p\end{array}$ & SNHG24 & 145 & -20.88 & 145 & -20.88 & 2175 & 22 & 1634 & 654 \\
\hline $\begin{array}{l}\text { hsa-miR-6754- } \\
5 p\end{array}$ & SNHG24 & 145 & -21.62 & 145 & -21.62 & 2177 & 22 & 1634 & 652 \\
\hline $\begin{array}{l}\text { hsa-miR-6798- } \\
5 p\end{array}$ & SNHG24 & 143 & -23.26 & 143 & -23.26 & 2265 & 23 & 1634 & 422 \\
\hline $\begin{array}{l}\text { hsa-miR-6805- } \\
3 p\end{array}$ & SNHG24 & 154 & -25.43 & 154 & -25.43 & 2280 & 23 & 1634 & 774 \\
\hline $\begin{array}{l}\text { hsa-miR-6812- } \\
5 p\end{array}$ & SNHG24 & 143 & -30.53 & 143 & -30.53 & 2293 & 25 & 1634 & 996 \\
\hline $\begin{array}{l}\text { hsa-miR-6815- } \\
5 p\end{array}$ & SNHG24 & 144 & -24.08 & 144 & -24.08 & 2299 & 23 & 1634 & 321 \\
\hline $\begin{array}{l}\text { hsa-miR-6818- } \\
3 p\end{array}$ & SNHG24 & 152 & -20.67 & 152 & -20.67 & 2306 & 22 & 1634 & 787 \\
\hline $\begin{array}{l}\text { hsa-miR-6819- } \\
5 p\end{array}$ & SNHG24 & 140 & -23.87 & 140 & -23.87 & 2307 & 22 & 1634 & 1002 \\
\hline $\begin{array}{l}\text { hsa-miR-6820- } \\
3 p\end{array}$ & SNHG24 & 147 & -21.1 & 147 & -21.1 & 2310 & 22 & 1634 & 1415 \\
\hline $\begin{array}{l}\text { hsa-miR-6847- } \\
5 p\end{array}$ & SNHG24 & 321 & -45.97 & 165 & -24.03 & 2363 & 23 & 1634 & 281471 \\
\hline $\begin{array}{l}\text { hsa-miR-6848- } \\
3 p\end{array}$ & SNHG24 & 140 & -20.26 & 140 & -20.26 & 2366 & 19 & 1634 & 953 \\
\hline $\begin{array}{l}\text { hsa-miR-6852- } \\
5 p\end{array}$ & SNHG24 & 149 & -29.16 & 149 & -29.16 & 2373 & 21 & 1634 & 39 \\
\hline
\end{tabular}




\begin{tabular}{|c|c|c|c|c|c|c|c|c|c|}
\hline Seq1 & Seq2 & $\begin{array}{l}\text { Tot } \\
\text { Score }\end{array}$ & $\begin{array}{l}\text { Tot } \\
\text { Energy }\end{array}$ & $\begin{array}{l}\text { Max } \\
\text { Score }\end{array}$ & $\begin{array}{l}\text { Max } \\
\text { Energy }\end{array}$ & Strand & Len1 & Len2 & Positions \\
\hline $\begin{array}{l}\text { hsa-miR-6861- } \\
3 p\end{array}$ & SNHG24 & 140 & -20.85 & 140 & -20.85 & 2393 & 18 & 1634 & 1453 \\
\hline $\begin{array}{l}\text { hsa-miR-6865- } \\
5 p\end{array}$ & SNHG24 & 142 & -20.95 & 142 & -20.95 & 2399 & 23 & 1634 & 322 \\
\hline $\begin{array}{l}\text { hsa-miR-6866- } \\
5 p\end{array}$ & SNHG24 & 145 & -21.27 & 145 & -21.27 & 2401 & 23 & 1634 & 503 \\
\hline $\begin{array}{l}\text { hsa-miR-6873- } \\
5 p\end{array}$ & SNHG24 & 163 & -26.55 & 163 & -26.55 & 2415 & 22 & 1634 & 424 \\
\hline $\begin{array}{l}\text { hsa-miR-6874- } \\
5 p\end{array}$ & SNHG24 & 143 & -25.2 & 143 & -25.2 & 2417 & 23 & 1634 & 74 \\
\hline $\begin{array}{l}\text { hsa-miR-6893- } \\
5 p\end{array}$ & SNHG24 & 145 & -21.66 & 145 & -21.66 & 2455 & 21 & 1634 & 673 \\
\hline $\begin{array}{l}\text { hsa-miR-7107- } \\
3 p\end{array}$ & SNHG24 & 153 & -22.93 & 153 & -22.93 & 2464 & 27 & 1634 & 860 \\
\hline $\begin{array}{l}\text { hsa-miR-7157- } \\
3 p\end{array}$ & SNHG24 & 305 & -42.06 & 155 & -21.29 & 2493 & 22 & 1634 & 519608 \\
\hline $\begin{array}{l}\text { hsa-miR- } \\
1273 \text { h-3p }\end{array}$ & SNHG24 & 150 & -22.22 & 150 & -22.22 & 2515 & 22 & 1634 & 837 \\
\hline hsa-miR-7974 & SNHG24 & 143 & -22.14 & 143 & -22.14 & 2533 & 24 & 1634 & 351 \\
\hline hsa-miR-8087 & SNHG24 & 146 & -27.19 & 146 & -27.19 & 2573 & 23 & 1634 & 473 \\
\hline $\begin{array}{l}\text { hsa-miR- } \\
\text { 10394-5p }\end{array}$ & SNHG24 & 152 & -22.84 & 152 & -22.84 & 2596 & 24 & 1634 & 770 \\
\hline $\begin{array}{l}\text { hsa-miR- } \\
\text { 10394-3p }\end{array}$ & SNHG24 & 141 & -21.6 & 141 & -21.6 & 2597 & 23 & 1634 & 1001 \\
\hline $\begin{array}{l}\text { hsa-miR- } \\
12115\end{array}$ & SNHG24 & 145 & -20.03 & 145 & -20.03 & 2636 & 24 & 1634 & 916 \\
\hline $\begin{array}{l}\text { hsa-miR- } \\
12117\end{array}$ & SNHG24 & 170 & -21.95 & 170 & -21.95 & 2638 & 20 & 1634 & 921 \\
\hline $\begin{array}{l}\text { hsa-miR- } \\
12122\end{array}$ & SNHG24 & 150 & -23.83 & 150 & -23.83 & 2643 & 25 & 1634 & 31 \\
\hline
\end{tabular}


Table 7

Prediction of mRNA-miRNA interaction pairs

\begin{tabular}{|c|c|c|c|c|c|c|c|c|c|}
\hline Seq1 & Seq2 & $\begin{array}{l}\text { Tot } \\
\text { Score }\end{array}$ & $\begin{array}{l}\text { Tot } \\
\text { Energy }\end{array}$ & $\begin{array}{l}\text { Max } \\
\text { Score }\end{array}$ & $\begin{array}{l}\text { Max } \\
\text { Energy }\end{array}$ & Strand & Len1 & Len2 & Positions \\
\hline $\begin{array}{l}\text { hsa-miR-149- } \\
3 p\end{array}$ & KISS1 & 151 & -29.06 & 151 & -29.06 & 480 & 21 & 143 & 35 \\
\hline $\begin{array}{l}\text { hsa-miR-328- } \\
5 p\end{array}$ & KISS1 & 299 & -66.55 & 159 & -37.7 & 704 & 23 & 143 & 5534 \\
\hline $\begin{array}{l}\text { hsa-miR-135b- } \\
3 p\end{array}$ & KISS1 & 144 & -24.63 & 144 & -24.63 & 728 & 22 & 143 & 48 \\
\hline $\begin{array}{l}\text { hsa-miR-486- } \\
3 p\end{array}$ & KISS1 & 151 & -23.4 & 151 & -23.4 & 848 & 21 & 143 & 112 \\
\hline $\begin{array}{l}\text { hsa-miR-92b- } \\
5 p\end{array}$ & FAM25A & 143 & -28.11 & 143 & -28.11 & 1133 & 22 & 56 & 5 \\
\hline hsa-miR-663a & KISS1 & 159 & -32.27 & 159 & -32.27 & 1412 & 22 & 143 & 65 \\
\hline hsa-miR-762 & KISS1 & 147 & -38.22 & 147 & -38.22 & 1534 & 22 & 143 & 64 \\
\hline $\begin{array}{l}\text { hsa-miR-675- } \\
5 p\end{array}$ & KISS1 & 151 & -22.73 & 151 & -22.73 & 1558 & 23 & 143 & 113 \\
\hline $\begin{array}{l}\text { hsa-miR-744- } \\
5 p\end{array}$ & KISS1 & 439 & -81.89 & 154 & -28.03 & 1618 & 22 & 143 & $\begin{array}{l}73113 \\
53\end{array}$ \\
\hline $\begin{array}{l}\text { hsa-miR-873- } \\
3 p\end{array}$ & FAM25A & 151 & -23.09 & 151 & -23.09 & 1637 & 22 & 56 & 13 \\
\hline $\begin{array}{l}\text { hsa-miR-1228- } \\
5 p\end{array}$ & KISS1 & 149 & -33.35 & 149 & -33.35 & 1732 & 21 & 143 & 72 \\
\hline $\begin{array}{l}\text { hsa-miR-1237- } \\
5 p\end{array}$ & KISS1 & 468 & -89.22 & 171 & -35.03 & 1752 & 21 & 143 & 606737 \\
\hline hsa-miR-1305 & KISS1 & 143 & -20.38 & 143 & -20.38 & 1826 & 22 & 143 & 15 \\
\hline hsa-miR-1275 & FAM25A & 141 & -25.74 & 141 & -25.74 & 1905 & 17 & 56 & 14 \\
\hline $\begin{array}{l}\text { hsa-miR-1908- } \\
5 p\end{array}$ & KISS1 & 314 & -52.63 & 159 & -26.58 & 1998 & 21 & 143 & 7057 \\
\hline hsa-miR-2110 & FAM25A & 154 & -27.34 & 154 & -27.34 & 2039 & 22 & 56 & 10 \\
\hline $\begin{array}{l}\text { hsa-miR-2115- } \\
5 p\end{array}$ & KISS1 & 143 & -23.35 & 143 & -23.35 & 2046 & 22 & 143 & 23 \\
\hline $\begin{array}{l}\text { hsa-miR-2682- } \\
5 p\end{array}$ & KISS1 & 154 & -26.11 & 154 & -26.11 & 2072 & 23 & 143 & 110 \\
\hline $\begin{array}{l}\text { hsa-miR-3127- } \\
5 p\end{array}$ & KISS1 & 152 & -26.39 & 152 & -26.39 & 2120 & 23 & 143 & 81 \\
\hline hsa-miR-3141 & KISS1 & 141 & -30.22 & 141 & -30.22 & 2168 & 19 & 143 & 68 \\
\hline $\begin{array}{l}\text { hsa-miR-3151- } \\
5 p\end{array}$ & KISS1 & 289 & -49.35 & 146 & -27.73 & 2200 & 21 & 143 & 11348 \\
\hline hsa-miR-3154 & FAM25A & 142 & -23.45 & 142 & -23.45 & 2213 & 22 & 56 & 6 \\
\hline
\end{tabular}




\begin{tabular}{|c|c|c|c|c|c|c|c|c|c|}
\hline Seq1 & Seq2 & $\begin{array}{l}\text { Tot } \\
\text { Score }\end{array}$ & $\begin{array}{l}\text { Tot } \\
\text { Energy }\end{array}$ & $\begin{array}{l}\text { Max } \\
\text { Score }\end{array}$ & $\begin{array}{l}\text { Max } \\
\text { Energy }\end{array}$ & Strand & Len1 & Len2 & Positions \\
\hline $\begin{array}{l}\text { hsa-miR-3180- } \\
3 p\end{array}$ & KISS1 & 288 & -53.98 & 148 & -29.34 & 2288 & 22 & 143 & 65109 \\
\hline $\begin{array}{l}\text { hsa-miR-3191- } \\
3 p\end{array}$ & KISS1 & 160 & -27.23 & 160 & -27.23 & 2330 & 23 & 143 & 54 \\
\hline hsa-miR-3196 & KISS1 & 292 & -46.19 & 152 & -25.88 & 2344 & 18 & 143 & 7162 \\
\hline hsa-miR-4254 & KISS1 & 140 & -23.26 & 140 & -23.26 & 2448 & 23 & 143 & 27 \\
\hline hsa-miR-4271 & FAM25A & 140 & -20.4 & 140 & -20.4 & 2483 & 19 & 56 & 10 \\
\hline hsa-miR-3665 & KISS1 & 176 & -36.81 & 176 & -36.81 & 2630 & 18 & 143 & 123 \\
\hline hsa-miR-3180 & KISS1 & 282 & -46.02 & 142 & -25.22 & 2706 & 19 & 143 & 68112 \\
\hline hsa-miR-4463 & FAM25A & 163 & -34.5 & 163 & -34.5 & 2951 & 17 & 56 & 17 \\
\hline hsa-miR-4466 & KISS1 & 156 & -25.99 & 156 & -25.99 & 2964 & 18 & 143 & 120 \\
\hline hsa-miR-4488 & KISS1 & 296 & -51.09 & 156 & -30.08 & 3028 & 18 & 143 & 7037 \\
\hline hsa-miR-3960 & KISS1 & 141 & -42.59 & 141 & -42.59 & 3156 & 20 & 143 & 69 \\
\hline $\begin{array}{l}\text { hsa-miR-4649- } \\
5 p\end{array}$ & KISS1 & 142 & -31.55 & 142 & -31.55 & 3220 & 24 & 143 & 62 \\
\hline hsa-miR-4651 & KISS1 & 286 & -62.95 & 144 & -32.75 & 3228 & 20 & 143 & 6173 \\
\hline $\begin{array}{l}\text { hsa-miR-4653- } \\
3 p\end{array}$ & KISS1 & 140 & -21.24 & 140 & -21.24 & 3236 & 23 & 143 & 85 \\
\hline hsa-miR-4656 & KISS1 & 158 & -35.67 & 158 & -35.67 & 3244 & 23 & 143 & 43 \\
\hline $\begin{array}{l}\text { hsa-miR-4667- } \\
5 p\end{array}$ & FAM25A & 143 & -20.76 & 143 & -20.76 & 3283 & 22 & 56 & 6 \\
\hline $\begin{array}{l}\text { hsa-miR-4690- } \\
5 p\end{array}$ & KISS1 & 140 & -25.99 & 140 & -25.99 & 3358 & 22 & 143 & 78 \\
\hline $\begin{array}{l}\text { hsa-miR-4697- } \\
5 p\end{array}$ & KISS1 & 293 & -51.18 & 150 & -27.45 & 3382 & 22 & 143 & 3065 \\
\hline $\begin{array}{l}\text { hsa-miR-4700- } \\
5 p\end{array}$ & FAM25A & 156 & -23.16 & 156 & -23.16 & 3391 & 22 & 56 & 11 \\
\hline hsa-miR-4706 & KISS1 & 151 & -27.53 & 151 & -27.53 & 3410 & 25 & 143 & 52 \\
\hline $\begin{array}{l}\text { hsa-miR-4725- } \\
3 p\end{array}$ & FAM25A & 153 & -27.83 & 153 & -27.83 & 3489 & 22 & 56 & 9 \\
\hline $\begin{array}{l}\text { hsa-miR-4728- } \\
5 p\end{array}$ & FAM25A & 149 & -27.02 & 149 & -27.02 & 3499 & 23 & 56 & 4 \\
\hline $\begin{array}{l}\text { hsa-miR-4728- } \\
5 p\end{array}$ & KISS1 & 149 & -24.92 & 149 & -24.92 & 3500 & 23 & 143 & 35 \\
\hline hsa-miR-4734 & KISS1 & 144 & -28.92 & 144 & -28.92 & 3520 & 22 & 143 & 75 \\
\hline hsa-miR-4739 & KISS1 & 166 & -33.97 & 166 & -33.97 & 3538 & 25 & 143 & 34 \\
\hline
\end{tabular}




\begin{tabular}{|c|c|c|c|c|c|c|c|c|c|}
\hline Seq1 & Seq2 & $\begin{array}{l}\text { Tot } \\
\text { Score }\end{array}$ & $\begin{array}{l}\text { Tot } \\
\text { Energy }\end{array}$ & $\begin{array}{l}\text { Max } \\
\text { Score }\end{array}$ & $\begin{array}{l}\text { Max } \\
\text { Energy }\end{array}$ & Strand & Len1 & Len2 & Positions \\
\hline $\begin{array}{l}\text { hsa-miR-4747- } \\
5 p\end{array}$ & FAM25A & 155 & -22.11 & 155 & -22.11 & 3567 & 22 & 56 & 7 \\
\hline $\begin{array}{l}\text { hsa-miR-4749- } \\
5 p\end{array}$ & KISS1 & 158 & -28.21 & 158 & -28.21 & 3574 & 22 & 143 & 52 \\
\hline $\begin{array}{l}\text { hsa-miR-4763- } \\
3 p\end{array}$ & KISS1 & 140 & -35.98 & 140 & -35.98 & 3632 & 24 & 143 & 67 \\
\hline $\begin{array}{l}\text { hsa-miR-4787- } \\
5 p\end{array}$ & KISS1 & 147 & -35.92 & 147 & -35.92 & 3718 & 22 & 143 & 66 \\
\hline $\begin{array}{l}\text { hsa-miR-5088- } \\
5 p\end{array}$ & KISS1 & 140 & -26.99 & 140 & -26.99 & 3836 & 24 & 143 & 43 \\
\hline hsa-miR-5090 & KISS1 & 148 & -23.21 & 148 & -23.21 & 3844 & 23 & 143 & 111 \\
\hline $\begin{array}{l}\text { hsa-miR-5196- } \\
5 p\end{array}$ & FAM25A & 150 & -25.61 & 150 & -25.61 & 3879 & 22 & 56 & 7 \\
\hline hsa-miR-5100 & KISS1 & 151 & -25.04 & 151 & -25.04 & 3896 & 22 & 143 & 98 \\
\hline $\begin{array}{l}\text { hsa-miR-664b- } \\
5 p\end{array}$ & KISS1 & 165 & -31.07 & 165 & -31.07 & 3916 & 24 & 143 & 42 \\
\hline hsa-miR-5698 & FAM25A & 148 & -24.28 & 148 & -24.28 & 4027 & 22 & 56 & 7 \\
\hline hsa-miR-5787 & KISS1 & 142 & -37.64 & 142 & -37.64 & 4056 & 20 & 143 & 71 \\
\hline hsa-miR-6073 & KISS1 & 142 & -21.14 & 142 & -21.14 & 4072 & 20 & 143 & 111 \\
\hline hsa-miR-6086 & FAM25A & 141 & -28.17 & 141 & -28.17 & 4097 & 20 & 56 & 14 \\
\hline hsa-miR-6090 & KISS1 & 145 & -41.3 & 145 & -41.3 & 4104 & 19 & 143 & 69 \\
\hline hsa-miR-6127 & KISS1 & 144 & -30.3 & 144 & -30.3 & 4112 & 19 & 143 & 40 \\
\hline hsa-miR-6133 & KISS1 & 140 & -24.97 & 140 & -24.97 & 4126 & 19 & 143 & 40 \\
\hline $\begin{array}{l}\text { hsa-miR-6499- } \\
5 p\end{array}$ & KISS1 & 140 & -21.39 & 140 & -21.39 & 4132 & 22 & 143 & 124 \\
\hline $\begin{array}{l}\text { hsa-miR-6500- } \\
5 p\end{array}$ & KISS1 & 141 & -24.94 & 141 & -24.94 & 4140 & 24 & 143 & 22 \\
\hline $\begin{array}{l}\text { hsa-miR-6727- } \\
5 p\end{array}$ & KISS1 & 147 & -33.54 & 147 & -33.54 & 4248 & 23 & 143 & 73 \\
\hline $\begin{array}{l}\text { hsa-miR-6752- } \\
5 p\end{array}$ & KISS1 & 292 & -61.54 & 147 & -32.47 & 4346 & 22 & 143 & 5932 \\
\hline $\begin{array}{l}\text { hsa-miR-6754- } \\
5 p\end{array}$ & KISS1 & 144 & -26.64 & 144 & -26.64 & 4354 & 22 & 143 & 39 \\
\hline $\begin{array}{l}\text { hsa-miR-6756- } \\
5 p\end{array}$ & KISS1 & 160 & -40.95 & 160 & -40.95 & 4362 & 23 & 143 & 68 \\
\hline $\begin{array}{l}\text { hsa-miR-6763- } \\
5 p\end{array}$ & FAM25A & 149 & -30.68 & 149 & -30.68 & 4389 & 19 & 56 & 11 \\
\hline
\end{tabular}




\begin{tabular}{|c|c|c|c|c|c|c|c|c|c|}
\hline Seq1 & Seq2 & $\begin{array}{l}\text { Tot } \\
\text { Score }\end{array}$ & $\begin{array}{l}\text { Tot } \\
\text { Energy }\end{array}$ & $\begin{array}{l}\text { Max } \\
\text { Score }\end{array}$ & $\begin{array}{l}\text { Max } \\
\text { Energy }\end{array}$ & Strand & Len1 & Len2 & Positions \\
\hline $\begin{array}{l}\text { hsa-miR-6775- } \\
5 p\end{array}$ & KISS1 & 292 & -64.84 & 147 & -42.3 & 4438 & 25 & 143 & 78110 \\
\hline $\begin{array}{l}\text { hsa-miR-6779- } \\
5 p\end{array}$ & KISS1 & 140 & -27.54 & 140 & -27.54 & 4454 & 21 & 143 & 35 \\
\hline $\begin{array}{l}\text { hsa-miR- } \\
6780 a-5 p\end{array}$ & KISS1 & 140 & -30.15 & 140 & -30.15 & 4458 & 23 & 143 & 40 \\
\hline $\begin{array}{l}\text { hsa-miR-6785- } \\
5 p\end{array}$ & FAM25A & 143 & -29.58 & 143 & -29.58 & 4477 & 22 & 56 & 4 \\
\hline $\begin{array}{l}\text { hsa-miR-6785- } \\
5 p\end{array}$ & KISS1 & 149 & -23.04 & 149 & -23.04 & 4478 & 22 & 143 & 37 \\
\hline $\begin{array}{l}\text { hsa-miR-6787- } \\
5 p\end{array}$ & KISS1 & 313 & -52.91 & 161 & -26.82 & 4486 & 22 & 143 & 5471 \\
\hline $\begin{array}{l}\text { hsa-miR-6796- } \\
5 p\end{array}$ & KISS1 & 140 & -22.78 & 140 & -22.78 & 4522 & 23 & 143 & 73 \\
\hline $\begin{array}{l}\text { hsa-miR-6803- } \\
5 p\end{array}$ & KISS1 & 155 & -44.31 & 155 & -44.31 & 4550 & 22 & 143 & 67 \\
\hline $\begin{array}{l}\text { hsa-miR-6805- } \\
5 p\end{array}$ & KISS1 & 150 & -25.79 & 150 & -25.79 & 4558 & 22 & 143 & 35 \\
\hline $\begin{array}{l}\text { hsa-miR-6810- } \\
5 p\end{array}$ & KISS1 & 140 & -23.43 & 140 & -23.43 & 4578 & 23 & 143 & 51 \\
\hline $\begin{array}{l}\text { hsa-miR-6813- } \\
5 p\end{array}$ & FAM25A & 144 & -26.88 & 144 & -26.88 & 4589 & 23 & 56 & 2 \\
\hline $\begin{array}{l}\text { hsa-miR-6814- } \\
5 p\end{array}$ & KISS1 & 140 & -23.38 & 140 & -23.38 & 4594 & 22 & 143 & 90 \\
\hline $\begin{array}{l}\text { hsa-miR-6816- } \\
5 p\end{array}$ & KISS1 & 307 & -55.38 & 154 & -29.08 & 4602 & 21 & 143 & 7260 \\
\hline $\begin{array}{l}\text { hsa-miR-6824- } \\
5 p\end{array}$ & KISS1 & 144 & -26.23 & 144 & -26.23 & 4634 & 22 & 143 & 41 \\
\hline $\begin{array}{l}\text { hsa-miR-6825- } \\
5 p\end{array}$ & FAM25A & 142 & -26.35 & 142 & -26.35 & 4637 & 22 & 56 & 4 \\
\hline $\begin{array}{l}\text { hsa-miR- } \\
6780 \mathrm{~b}-5 \mathrm{p}\end{array}$ & FAM25A & 156 & -29.83 & 156 & -29.83 & 4681 & 23 & 56 & 4 \\
\hline hsa-miR-6860 & FAM25A & 146 & -35.29 & 146 & -35.29 & 4781 & 22 & 56 & 8 \\
\hline $\begin{array}{l}\text { hsa-miR-6874- } \\
5 p\end{array}$ & KISS1 & 152 & -24.31 & 152 & -24.31 & 4834 & 23 & 143 & 23 \\
\hline $\begin{array}{l}\text { hsa-miR-6883- } \\
5 p\end{array}$ & FAM25A & 146 & -26.98 & 146 & -26.98 & 4869 & 22 & 56 & 4 \\
\hline $\begin{array}{l}\text { hsa-miR-6883- } \\
5 p\end{array}$ & KISS1 & 152 & -27.09 & 152 & -27.09 & 4870 & 22 & 143 & 37 \\
\hline $\begin{array}{l}\text { hsa-miR-6884- } \\
5 p\end{array}$ & FAM25A & 144 & -20.72 & 144 & -20.72 & 4873 & 22 & 56 & 13 \\
\hline
\end{tabular}




\begin{tabular}{|llllllllll|}
\hline Seq1 & Seq2 & $\begin{array}{l}\text { Tot } \\
\text { Score }\end{array}$ & $\begin{array}{l}\text { Tot } \\
\text { Energy }\end{array}$ & $\begin{array}{l}\text { Max } \\
\text { Score }\end{array}$ & $\begin{array}{l}\text { Max } \\
\text { Energy }\end{array}$ & Strand & Len1 & Len2 & Positions \\
\hline $\begin{array}{l}\text { hsa-miR-6885- } \\
5 p\end{array}$ & KISS1 & 289 & -47.02 & 149 & -25.56 & 4878 & 25 & 143 & 5530 \\
\hline $\begin{array}{l}\text { hsa-miR-7109- } \\
5 p\end{array}$ & KISS1 & 143 & -22.81 & 143 & -22.81 & 4934 & 21 & 143 & 62 \\
$\begin{array}{l}\text { hsa-miR-7110- } \\
5 p\end{array}$ & KISS1 & 145 & -29.07 & 145 & -29.07 & 4938 & 21 & 143 & 67 \\
\hline $\begin{array}{l}\text { hsa-miR-8072 } \\
\text { hsa-miR-8089 }\end{array}$ & KISS1 & 145 & -35.26 & 145 & -35.26 & 5116 & 20 & 143 & 60 \\
\hline $\begin{array}{l}\text { hsa-miR- } \\
\text { Fa394-3p }\end{array}$ & KISS1 & 159 & -30.91 & 159 & -30.91 & 5149 & 24 & 56 & 7 \\
\hline $\begin{array}{l}\text { hsa-miR- } \\
10396 a-5 p\end{array}$ & KISS1 & 294 & -29.43 & 144 & -29.43 & 5194 & 23 & 143 & 119 \\
\hline $\begin{array}{l}\text { hsa-miR- } \\
\text { 10396b-5p }\end{array}$ & KISS1 & 158 & -25.72 & 158 & -25.72 & 5224 & 20 & 143 & 65 \\
\hline
\end{tabular}

\section{Construction of ceRNA network}

Based on the interaction pairs of IncRNA-miRNA, mRNA-miRNA, and IncRNA-mRNA, a ceRNA network was constructed with 11 miRNAs, one IncRNA, and one mRNA, including SNHG24-has-miR-4739-KISS1, SNHG24-hasmiR-4734-KISS1, and SNHG24-has-miR-135b-3p-KISS1 (Fig. 8). These RNA interactions may contribute to the progression and metastasis of pancreatic cancer.

\section{GO functional and pathway enrichment analysis in ceRNA network}

Based on the IncRNA-mRNA interaction pairs in the ceRNA network, KEGG pathway annotation was performed. The results revealed that SNHG24 mainly correlated with $\mathrm{GnRH}$ secretion and neuroactive ligand-receptor interaction in pancreatic cancer (Fig. 9).

\section{Discussion}

Pancreatic cancer is the most malignant tumor of the digestive system, and it is also known as the 'king of cancers' [24]. According to the latest statistical data, the incidence of pancreatic cancer ranks eighth among all malignant tumors in China, and the cumulative 5-year survival rate is only 7.2\% [25]. Although growing number of studies have focused on dissecting and evaluating the underlying molecular etiology of pancreatic cancer over the past few years, the precise mechanisms of pancreatic cancer initiation and development are still poorly understood. In our study, eight DEsnoRNAs and 93 trend-related snoRNAs were identified. Ten host genes of snoRNAs were also identified, and functional analyses revealed that the ten host genes were significantly enriched in $\mathrm{GO}$ terms including mitotic chromosome condensation and endocytosis pathway. SNORA38B was found to be associated with survival and prognosis. SNORD17 and SNORA11 were found to be negatively correlated with methylation. Two trend-related SNHGs were also extracted. Additionally, a ceRNA network was constructed with the 11 miRNAs, one IncRNAs, and one mRNA. SNHG24 was mainly correlated with $\mathrm{GnRH}$ secretion and neuroactive ligand-receptor interaction in pancreatic cancer. 
SNORD17, also known as HBI-43, has been shown to be involved in the differentiation of different cancer subtypes [26]. A recent report showed that SNORD17 was functionally related to a specific active gene TRIM25 [27]. Wang et al. [28] demonstrated that the control of TRIM25 RNA by an interplay between IGF2BP3 and miR-3614-3p represents a mechanism for breast cancer cell proliferation. More notably, Li et al. [29] reported that suppression of TRIM25 expression using high levels of miR-873 dictates MTA1 protein upregulation in hepatocellular carcinoma. Therefore, it is quite reasonable to speculate that as the regulator of TRIM25, the expression pattern of SNORD17 may also be sensitive to distinguish the different cancer subtypes. Interestingly, Davanian et al. [30] first identified SNORA11 as a distinct ncRNA signature of ameloblastoma. Herein, our methylation correlation analysis showed that SNORD17 and SNORA11 were also negatively correlated with methylation. Taken together, our results suggest that SNORD17 and SNORA11 are likely associated with pancreatic cancer occurrence and progression. In addition, our functional analysis showed that SNORD17 participated in some BPs, especially in endocytosis. Numerous studies have reported that endocytosis serves as a key regulatory mechanism in carcinogenesis [31, 32], which further implies that the molecular interactions between SNORD17 and endocytosis may be a novel therapeutic target for pancreatic cancer treatment. In this study, we also found SNORA38B to be associated with survival and prognosis of pancreatic cancer. Until now, SNORA38B has not been associated with any other neoplasms, thus making it unique in this respect.

Clorf79, also known as SNHG12, is a novel IncRNA identified to be upregulated in several cancer cells, such as human osteosarcoma cell [33], nasopharyngeal carcinoma cell [34], and human endometrial carcinoma [35]. Zhao et al. [36] verified that SNHG12 promotes angiogenesis after ischemic stroke through the miR-150/VEGF pathway, that further clarified the mechanism of angiogenesis after ischemic stroke, and provided a target for the treatment of ischemic stroke. Wang et al. [37] suggested that SNHG12 contributes to the oncogenic potential of triple-negative breast cancer and may be a promising therapeutic target. Wang et al. [38] illustrated that SNHG12 promoted cell growth and inhibited cell apoptosis in CRC cells, indicating that SNHG12 might be a useful biomarker for colorectal cancer. In the current study, C1orf79 was identified as the host gene of DEsnoRNAs and trend-related snoRNAs. C1orf79 is a new biomarker that to the best of our knowledge has not yet been reported to be associated with pancreatic cancer.

SNHG24, a prominent regulator of the ceRNA network, was found to be closely linked with the process of GnRH secretion and neuroactive ligand-receptor interaction, and exhibited significant correlation with KISS1. Reports suggest that the IncRNA SNHG24 can regulate TNF-a expression, and TNF-a and its associated IncRNA SNHG24 represent potential therapeutic targets for bone-invasive pituitary adenomas in the future [39]. Eckstein et al. [40] highlighted a strong correlation between $\mathrm{GnRH}$ secretion and the risk of prostate cancer, which directly supports our findings. Reports suggest that the pathway of neuroactive ligand-receptor interaction is associated with a variety of cancers [41], which further implies that the molecular interactions between SNHG24, GnRH secretion, and neuroactive ligand-receptor interaction may be a novel therapeutic target for pancreatic cancer treatment. In addition, Wang et al. [42] showed that an increase in the expression of KiSS1 could inhibit invasion of pancreatic cancer cells without affecting cell proliferation. McNally et al. [43] suggested that the induction of KISS1 expression has the potential of adjuvant therapy in pancreatic cancer. More interestingly, the ceRNA-related with SNHG24-KISS1 network analysis found that SNHG24-KISS1 had a strong relationship with miRNAs such as has-miR-4739, has-miR-4734, and has-miR-135b-3p. Increasing evidence suggest that miRNA serve as functional modulators in tumor development $[44,45]$. Taken together, we speculate that miRNAs such as has-miR-4739, has-miR-4734, and has-miR135b-3p contribute to pancreatic cancer progression possibly by targeting the SNHG24-KISS1 axis, which still needs to be confirmed through further bioinformatics analysis and experimental evidence. 
Although we explored the potential molecular mechanisms of pancreatic cancer occurrence and development using a bioinformatics approach, there were still several limitations in this study. Firstly, no DESNHGs were obtained in this study. In addition, relevant experiments including cell biology assays, and animal and clinical studies need to be performed to verify the multiple candidate targets and signaling pathways identified from our bioinformatics analyses.

\section{Conclusion}

The snoRNAs and SNHGs identified in our study may serve as potential markers for the early detection of pancreatic cancer. However, our findings still need to be validated through further studies.

\section{Abbreviations}

\begin{tabular}{ll} 
Abbreviation & Full name \\
\hline snoRNAs & Small nucleolar noncoding RNAs \\
\hline SNHG & Small nucleolar RNA host gene \\
\hline DEsnoRNAs & Differentially expressed snoRNAs \\
\hline DESNHGs & Differentially expressed SNHGs \\
\hline TCGA & The cancer genome atlas \\
\hline KEGG & Kyoto Encyclopedia of Genes and Genomes \\
\hline GLOBOCAN & Global cancer epidemic statistics \\
\hline IncRNAs & Long noncoding RNAs \\
\hline ceRNAs & Competing endogenous RNAs \\
\hline BP & Biological process \\
\hline CC & Cellular components \\
\hline MF & Molecular function \\
\hline GO & Gene ontology
\end{tabular}

\section{Declarations}

\section{Ethics approval and consent to participate}

Not Applicable.

\section{Consent for publication}

Not Applicable.

\section{Availability of data and materials}

The datasets generated during the current study are not publicly available but obtained from corresponding authors on reasonable request. 


\section{Competing Interests}

The authors declare that no conflicts of interest exist.

\section{Funding}

This work was supported by the Key Laboratory of diagnosis, treatment and research of digestive system tumor of Zhejiang Province(2019E10020),the Natural public welfare fund of Zhejiang Province (LGC20H160002), the Medical and health science and technology Foundation of Zhejiang Province(2019KY595, 2018KY690, 2018KY699, 2017KY593,2017KY594)and the Natural Science Foundation of Ningbo(2018A610368, 2017A610145, 2017A610158, 2016A610135).

\section{Authors' Contributions}

All authors participated in the conception and design of the study;

Conceived and drafted the manuscript: Shuwen Han and Xi Yang ;

Analyzed the data: Siqi Dai ,Yangyang Xie , Xi Yang and Xiaoyu Dai ;

Wrote the paper:Shuwen Han and Yangyang Xie;

All authors read and approved the paper.

\section{Acknowledgements}

Thanks for the data support provided by the TCGA database

\section{References}

[1] Ilic M, Ilic I (2016). Epidemiology of pancreatic cancer. World J Gastroenterol, 22:9694-9705.

[2] Mcguire S (2016). World Cancer Report 2014. Geneva, Switzerland: World Health Organization, International Agency for Research on Cancer, WHO Press, 2015. Advances in Nutrition, 7:418-419.

[3] Bray F, Ferlay J, Soerjomataram I, al e (2018). Global Cancer Statistics 2018: GLOBOCAN Estimates of Incidence and Mortality Worldwide for 36 Cancers in 185 Countries. CA Cancer J Clin, 68:394-424.

[4] Conroy T, Van Laethem J-L (2019). Combination or single-agent chemotherapy as adjuvant treatment for pancreatic cancer? The Lancet Oncology.

[5] Boeck S, Heinemann V Improving post-surgical management of resected pancreatic cancer. Lancet, 390:847-848.

[6] Are C, Chowdhury S, Ahmad H, Ravipati A, Song T, Shrikandhe S, et al. Predictive global trends in the incidence and mortality of pancreatic cancer based on geographic location, socio-economic status, and demographic shift. Journal of Surgical Oncology.

[7] Xu B, Liu J, Xiang X, Liu S, Zhong P, Xie F, et al. (2018). Expression of miRNA-143 in Pancreatic Cancer and Its Clinical Significance. Cancer Biother Radiopharm, 33:373-379. 
[8] Li X, Deng SJ, Zhu S, Jin Y, Cui SP, Chen JY, et al. (2016). Hypoxia-induced IncRNA-NUTF2P3-001 contributes to tumorigenesis of pancreatic cancer by derepressing the miR-3923/KRAS pathway. Oncotarget, 7:6000-6014.

[9] Qadir MI, Faheem A (2017). miRNA: A Diagnostic and Therapeutic Tool for Pancreatic Cancer. Crit Rev Eukaryot Gene Expr, 27:197-204.

[10] Bhan A, Soleimani M, Mandal SS (2017). Long Noncoding RNA and Cancer: A New Paradigm. Cancer Res, 77:3965-3981.

[11] Fu Z, Chen C, Zhou Q, Wang Y, Zhao Y, Zhao X, et al. LncRNA HOTTIP modulates cancer stem cell properties in human pancreatic cancer by regulating HOXA9. Cancer Letters:S0304383517305694.

[12] Cheng D, Fan J, Ma Y, Zhou Y, Qin K, Shi M, et al. LncRNA SNHG7 promotes pancreatic cancer proliferation through ID4 by sponging miR-342-3p. Cell \& Bioscience, 9.

[13] Dong XY, Guo P, Boyd J, Sun X, Li Q, Zhou W, et al. (2009). Implication of snoRNA U50 in human breast cancer. J Genet Genomics, 36:447-454.

[14] Okugawa Y, Toiyama Y, Toden S, Mitoma H, Nagasaka T, Tanaka K, et al. (2017). Clinical significance of SNORA42 as an oncogene and a prognostic biomarker in colorectal cancer. Gut, 66:107-117.

[15] Popov A, Szabo A, Mandys V Small nucleolar RNA U91 is a new internal control for accurate microRNAs quantification in pancreatic cancer. Bmc Cancer, 15:774.

[16] Goldman M, Craft B, Zhu J, Haussler D (2019). The UCSC Xena system for cancer genomics data visualization and interpretation. Cancer Research, 77:326470.

[17] MD R, DJ M, GK S (2010). edgeR: a Bioconductor package for differential expression analysis of digital gene expression data. Bioinformatics, 26:139-140.

[18] Ritchie ME, Belinda P, Di W, Hu Y, Law CW, Wei S, et al. (2015). \n limma powers differential expression analyses for RNA-sequencing and microarray studies. Nucleic Acids Research:7.

[19] Ernst J, Bar-Joseph Z STEM: a tool for the analysis of short time series gene expression data. 7:191-190.

[20] Yoshihama M, Nakao A, Kenmochi N (2013). snOPY: a small nucleolar RNA orthological gene database. Bmc Research Notes, 6:1-5.

[21] Yu G, Wang L-G, Han Y, He Q-Y clusterProfiler: an R Package for Comparing Biological Themes Among Gene Clusters. OMICS-A JOURNAL OF INTEGRATIVE BIOLOGY, 16:284-287.

[22] Gong J, Li Y, Liu C-j, Xiang Y, Li C, Ye Y, et al. A Pan-cancer Analysis of the Expression and Clinical Relevance of Small Nucleolar RNAs in Human Cancer. Cell Reports, 21:1968-1981.

[23] Enright AJ, John B, Gaul U, TuschI T, Sander C, Marks DS (2003). MicroRNA targets in Drosophila. Genome Biol, 5:R1.

[24] Timaner M, Shaked Y (2019). Elucidating the roles of ASPM isoforms reveals a novel prognostic marker for pancreatic cancer. The Journal of Pathology. 
[25] Zeng H, Chen W, R Z (2018). Changing cancer survival in China during 2003-15: a pooled analysis of 17 population-based cancer registries. Lancet Global Health, 6:e555-e567.

[26] Pan X, Chen L (2019). Analysis of Expression Pattern of snoRNAs in Different Cancer Types with Machine Learning Algorithms. 20.

[27] Choudhury NR, Heikel G, Trubitsyna M, Kubik P, Nowak JS, Webb S, et al. (2017). RNA-binding activity of TRIM25 is mediated by its PRY/SPRY domain and is required for ubiquitination. 15:105.

[28] Wang Z, Tong D, Han C, Zhao Z, Wang X, Jiang T, et al. (2019). Blockade of miR-3614 maturation by IGF2BP3 increases TRIM25 expression and promotes breast cancer cell proliferation. EBioMedicine, 41:357-369.

[29] Li YH, Zhong M, Zang HL, Tian XF (2018). The E3 ligase for metastasis associated 1 protein, TRIM25, is targeted by microRNA-873 in hepatocellular carcinoma. Exp Cell Res, 368:37-41.

[30] Davanian H, Balasiddaiah A, Heymann R, Sundstrom M, Redenstrom P, Silfverberg M, et al. (2017).

Ameloblastoma RNA profiling uncovers a distinct non-coding RNA signature. Oncotarget, 8:4530-4542.

[31] Zhang Y, Zhang B (2008). TRAIL resistance of breast cancer cells is associated with constitutive endocytosis of death receptors 4 and 5. Mol Cancer Res, 6:1861-1871.

[32] Yeager A (2013). Investigation of caveolae-mediated endocytosis in response to interferon.

[33] Ruan W, Wang P, Feng S, Xue Y, Li Y (2016). Long non-coding RNA small nucleolar RNA host gene 12 (SNHG12) promotes cell proliferation and migration by upregulating angiomotin gene expression in human osteosarcoma cells. Tumour Biol, 37:4065-4073.

[34] Gong Z, Zhang S, Zeng Z, Wu H, Yang Q, Xiong F, et al. (2014). LOC401317, a p53-regulated long non-coding RNA, inhibits cell proliferation and induces apoptosis in the nasopharyngeal carcinoma cell line HNE2. PLoS One, 9:e110674.

[35] Zhai W, Li X, Wu S, Zhang Y, Pang H, Chen W (2015). Microarray expression profile of IncRNAs and the upregulated ASLNC04080 IncRNA in human endometrial carcinoma. Int J Oncol, 46:2125-2137.

[36] Zhao M, Wang J, Xi X, Tan N, Zhang L (2018). SNHG12 Promotes Angiogenesis Following Ischemic Stroke via Regulating miR-150/VEGF Pathway. Neuroscience, 390:231-240.

[37] Wang O, Yang F, Liu Y, Lv L, Ma R, Chen C, et al. (2017). C-MYC-induced upregulation of IncRNA SNHG12 regulates cell proliferation, apoptosis and migration in triple-negative breast cancer. Am J Transl Res, 9:533-545.

[38] Wang JZ, Xu CL, Wu H, Shen SJ (2017). LncRNA SNHG12 promotes cell growth and inhibits cell apoptosis in colorectal cancer cells. Braz J Med Biol Res, 50:e6079.

[39] Zhu H, Guo J, Shen Y, Dong W, Gao H, Miao Y, et al. (2018). Functions and Mechanisms of Tumor Necrosis Factor-alpha and Noncoding RNAs in Bone-Invasive Pituitary Adenomas. Clin Cancer Res, 24:5757-5766.

[40] Eckstein N, Haas B Clinical pharmacology and regulatory consequences of GnRH analogues in prostate cancer. European Journal of Clinical Pharmacology, 70:791-798. 
[41] Yang Z, Yan Z, Chen L. 2012. in silico identification of novel cancer-related genes by comparative genomics of naked mole rat and rat.

[42] Wang CH, Qiao C, Wang RC, Zhou WP (2016). KiSS1 mediated suppression of the invasive ability of human pancreatic carcinoma cells is not dependent on the level of KiSS1 receptor GPR54. Mol Med Rep, 13:123-129.

[43] McNally LR, Welch DR, Beck BH, Stafford LJ, Long JW, Sellers JC, et al. (2010). KISS1 over-expression suppresses metastasis of pancreatic adenocarcinoma in a xenograft mouse model. Clin Exp Metastasis, 27:591600.

[44] Rathod SS, Rani SB, Khan M, Muzumdar D, Shiras A Tumor suppressive miRNA-34a suppresses cell proliferation and tumor growth of glioma stem cells by targeting Akt and Wnt signaling pathways. Febs Open Bio, 4:485-495.

[45] Fang RH, Ji XB (2018). [Advances in the research of the relationship between miRNA-29c and cancer]. Lin Chung Er Bi Yan Hou Tou Jing Wai Ke Za Zhi, 32:312-317.

\section{Figures}

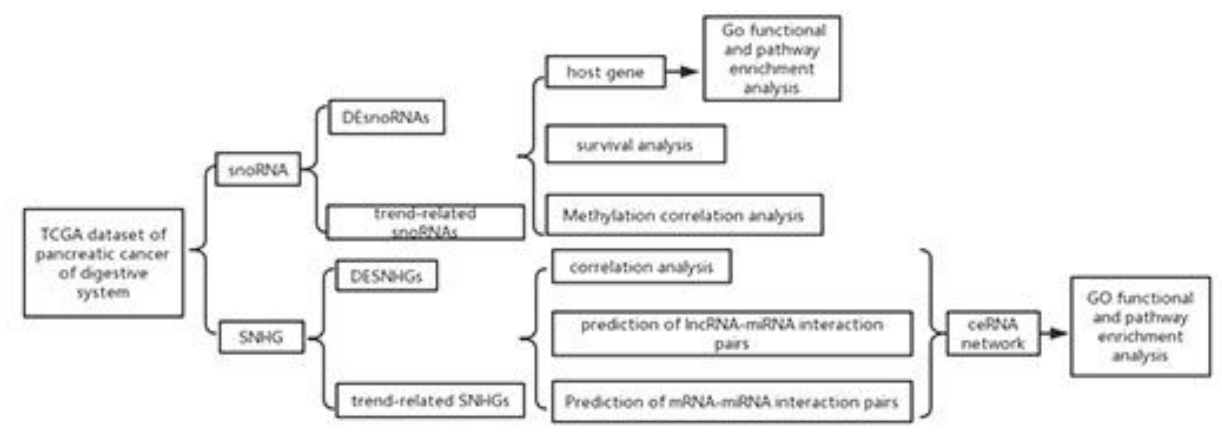

Figure 1

The flow chart of this study 
A

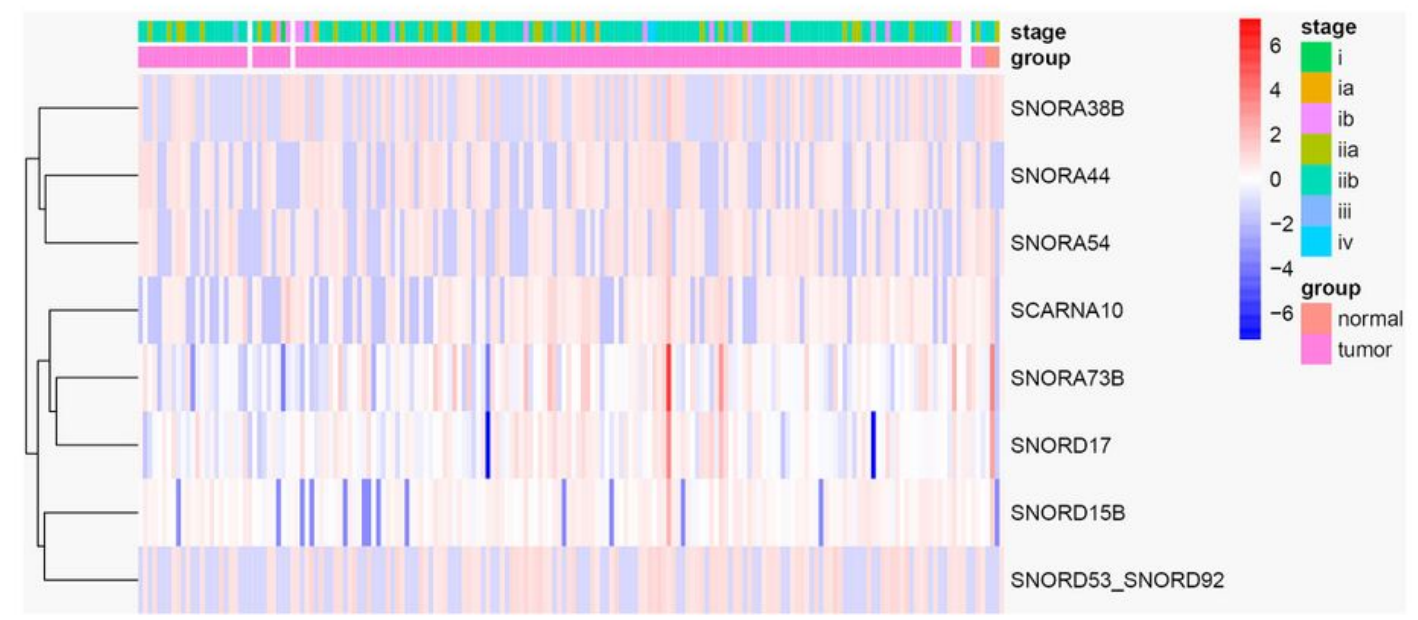

B

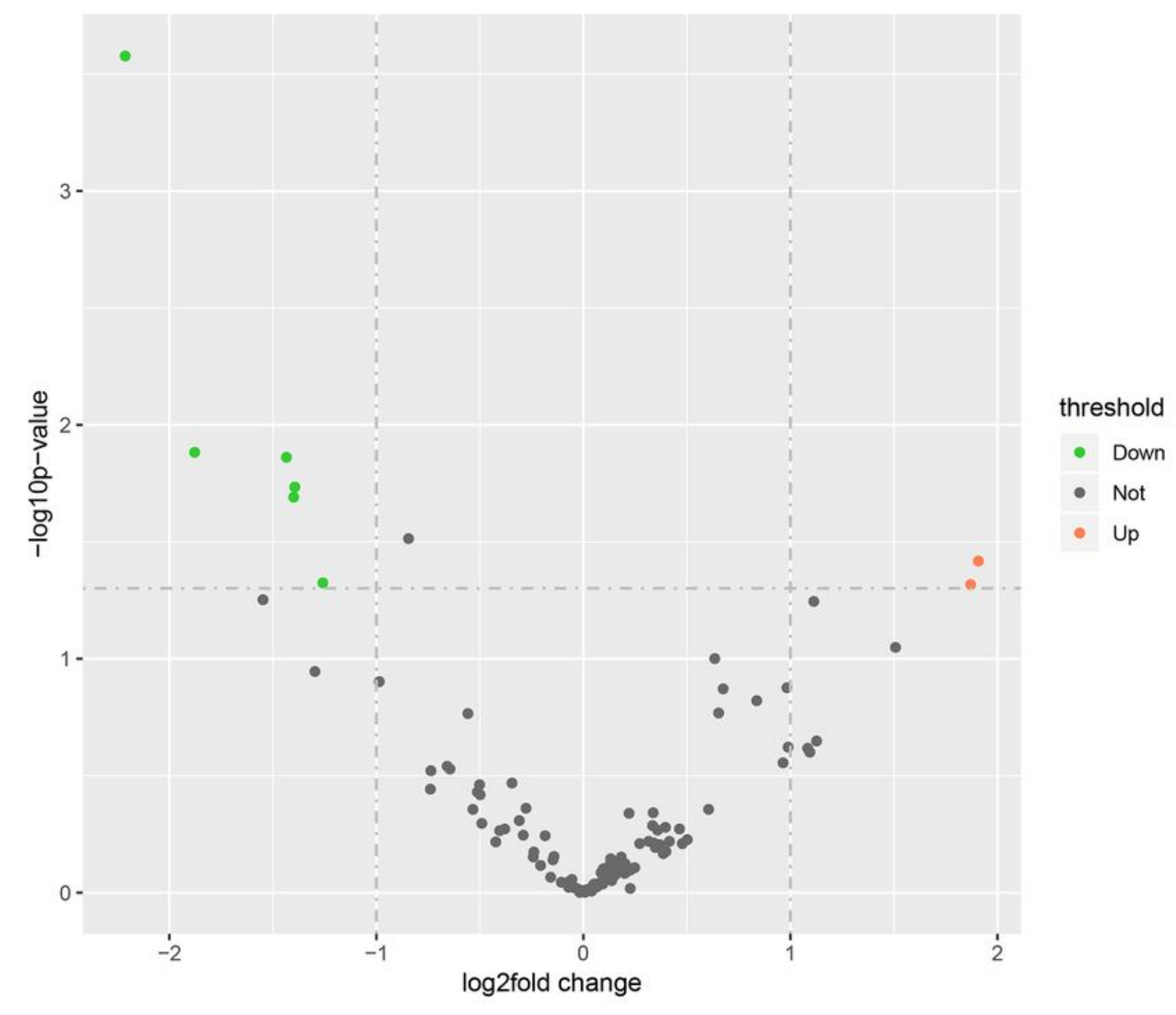

Figure 2

Identification of differentially expressed snoRNAs (DEsnoRNAs) and trend-related snoRNAs To screen the differentially expressed snoRNAs (DEsnoRNAs) between the tumor and normal groups based on the read count data of the snoRNAs, the TMM (trimmed mean of $\mathrm{m}$ values) normalization provided by the $\mathrm{R}$ software package edge was used to preprocess the read count. Then the quasi-likelihood (QL) F-test in the edgeR package was applied for screening the DEsnoRNAs, and the screening criteria was $p<0.05, \| \log F C \mid>1$ (foldchange $>1.2$ ). A total of eight DEsnoRNAs, including two upregulated and six downregulated snoRNAs were identified. A: Heatmap of DEsnoRNAs; B: volcano plot of DEsnoRNAs 
A
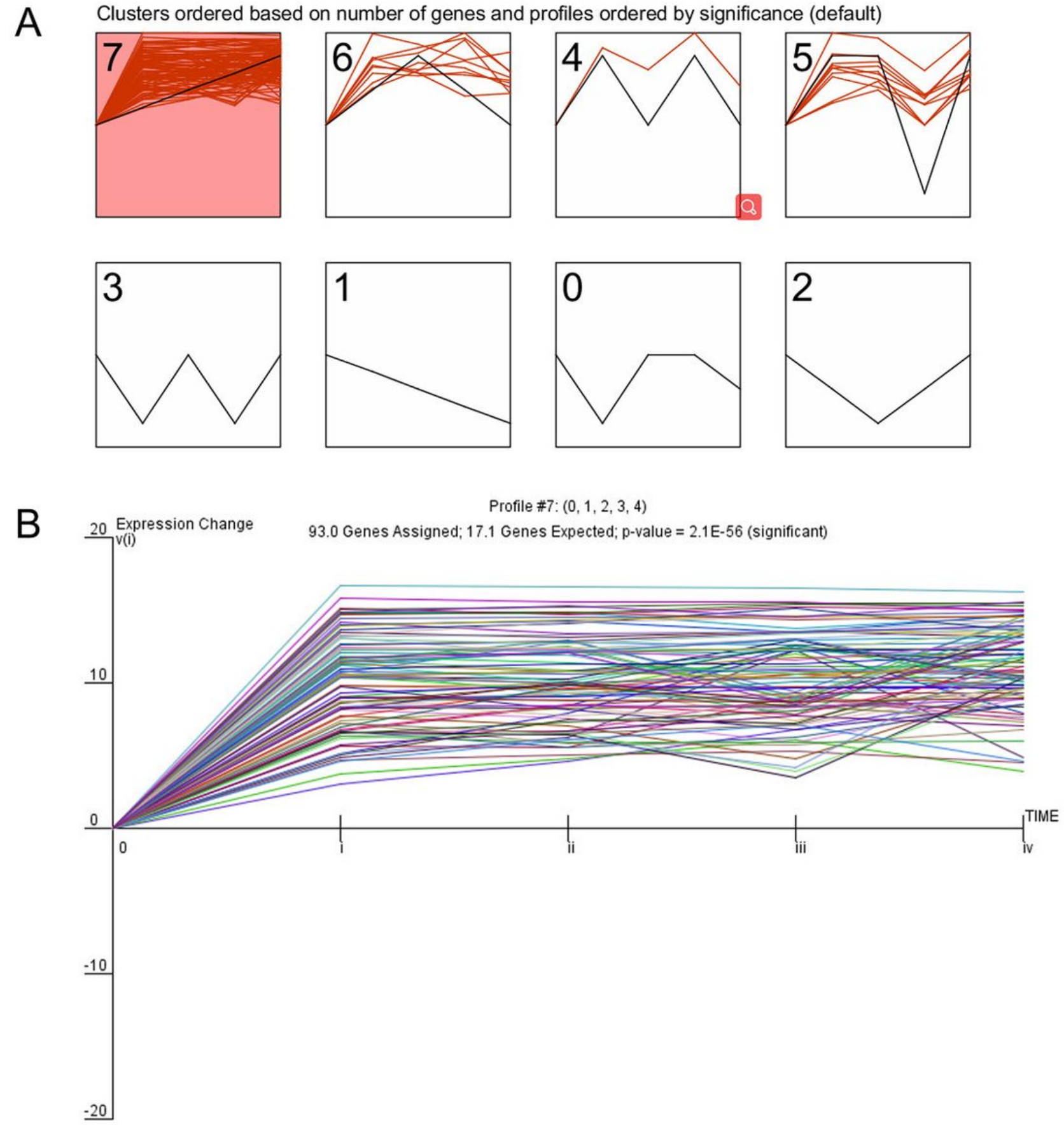

\section{Figure 3}

Identification of trend-related snoRNAs The trend-related snoRNAs were obtained based on the classification of tumor stage diagnosis, including stage I, stage II, stage II, and stage IV. The data was analyzed using the STEM software for trend analysis. The processing settings included with 'No normalization/add 0 ' and screened with $p<$ 0.05. A total of 93 trend-related snoRNAs were obtained. A: Sample diagram of trend analysis. The colored module in the figure represents significant trends with $p<0.05$. Each colored line represents the expression trend of a gene at different points with the black line representing the general trend of the changes of these genes. The colorless modules represent non-significant trends, and were excluded from the subsequent analyses. B: Sample diagram of a module. A single module in the sample graph of the trend analysis is magnified to show the expression of each gene according to the stage node. 


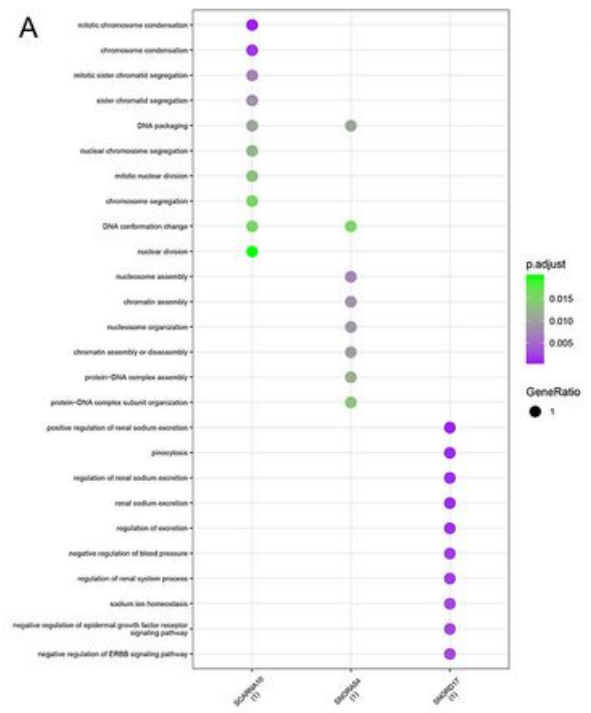

B

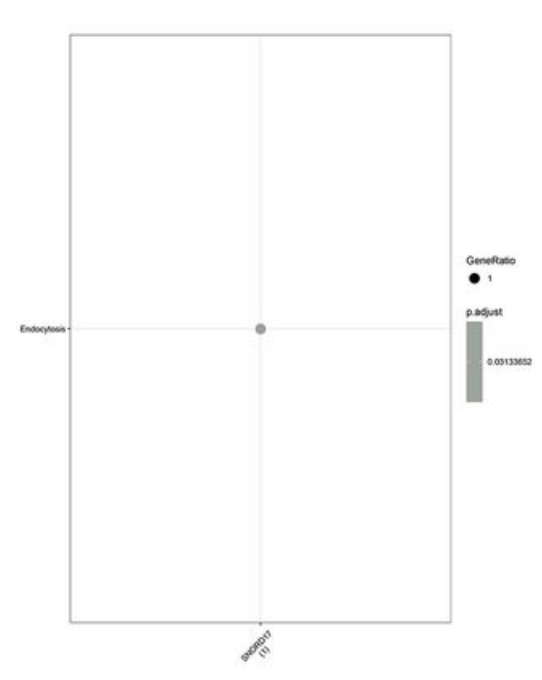

C

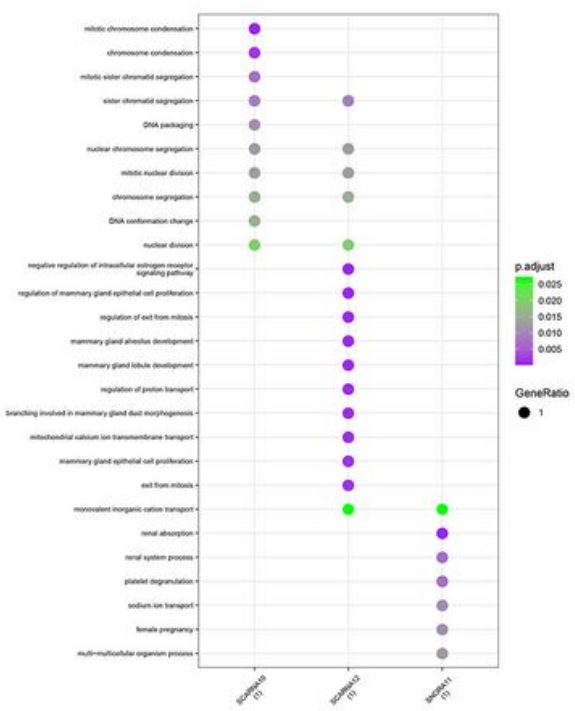

Figure 4

Functional analysis of host gene of DEsnoRNAs and trend-related snoRNAs To functionally analyze the host gene of DEsnoRNAs and trend-related snoRNAs, based on the target genes of the snoRNAs, the clusterprofiler tool was used to perform GO functional and KEGG pathway analyses. The functional analyses indicated that host genes of DEsnoRNAs were significantly enriched in several GO terms including mitotic chromosome condensation, retromer complex, and dopamine receptor binding, and endocytosis pathway, while host gene of trend-related snoRNAs were predominantly enriched in mitotic chromosome condensation, presynaptic active zone, and sphingolipid binding. A: The Gene ontology (GO) functional analysis of host gene of DEsnoRNAs; B: The Kyoto Encyclopedia of Genes and Genomes (KEGG) pathway enrichment analysis of host gene of DEsnoRNAs; C: The Gene ontology (GO) functional analysis of top 10 host gene of trend-related snoRNAs. 

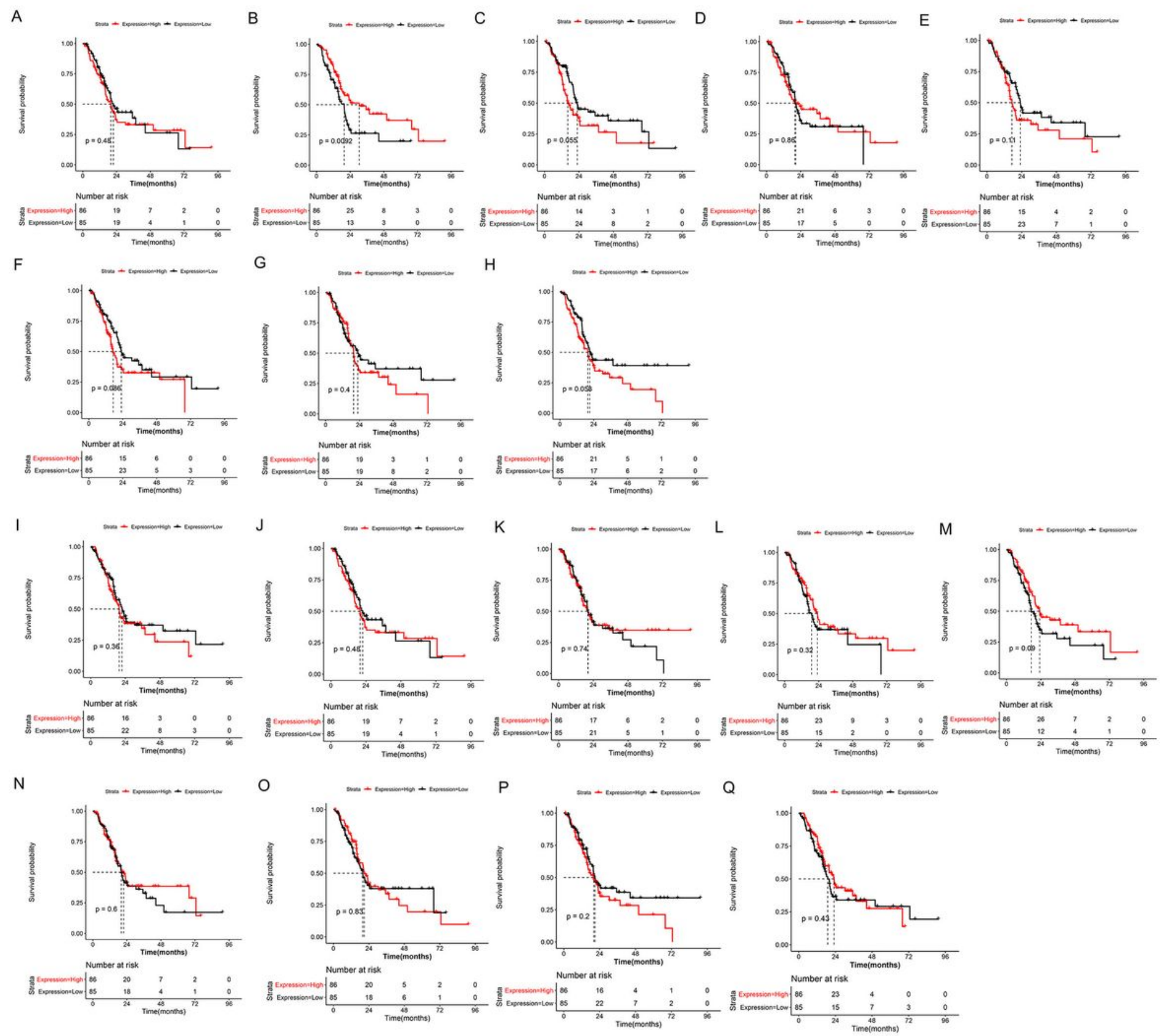

\section{Figure 5}

Survival analysis of DEsnoRNAs and trend-related snoRNAs To evaluate the association of the snoRNAs with survival and prognosis, the survival analysis is conducted on the DEsnoRNAs and trend-related snoRNAs data. $p<$ 0.05 was considered to be an association with survival and prognosis. SNORA38B, a DEsnoRNA with $p=0.0092$, was found to be associated with survival and prognosis. A: SCARNA10; B: SNORA38B; C: SNORA44; D: SNORA54; E: SNORA73B; F: SNORD15B; G: SNORD17; H: SNORD53_SNORD92; I: ACA59; J: SCARNA10; K: SCARNA12; L:SNORA11; M: SNORA12; N: SNORA13; O:SNORA14B; P: SNORA16B; Q: SNORA16 
A

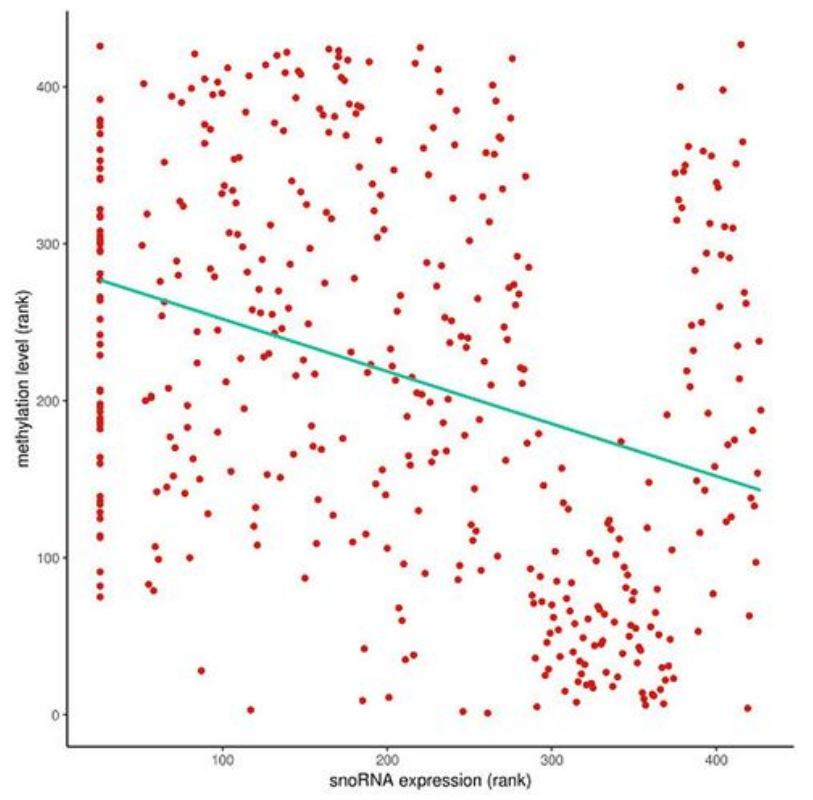

B

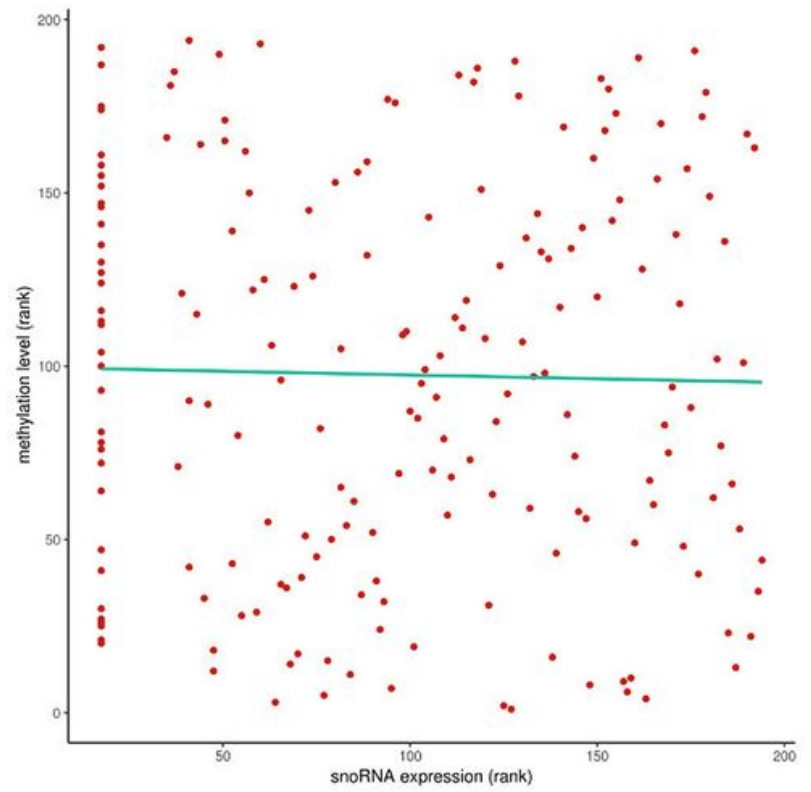

\section{Figure 6}

Methylation correlation analysis of DEsnoRNAs and trend-related snoRNAs To evaluate the association of the snoRNAs with methylation, methylation correlation analysis was performed using the SNORic tool. SNORD17 and SNORA11 were found to be negatively correlated with methylation. A: The methylation correlation analysis of DEsnoRNAs; B: The methylation correlation analysis of trend-related snoRNAs. The abscissa shows the expression value of the snoRNA sample, the ordinate shows the expression value of the methylated sample, and the blue line is the trend line. In general, a positive correlation has a positive coefficient, and a negative correlation has a negative coefficient. When the correlation coefficient is relatively large, the correlation is relatively strong and the points are more concentrated on both sides of the trend line.

A
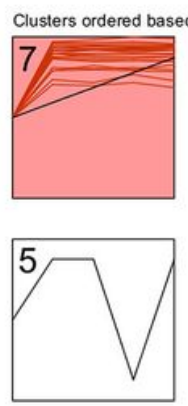
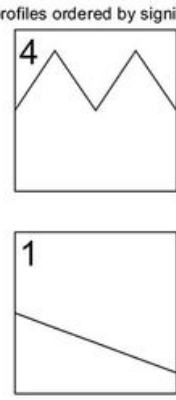
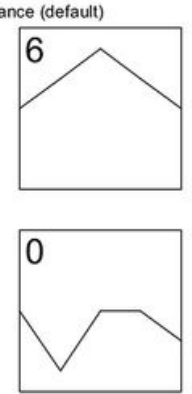

B

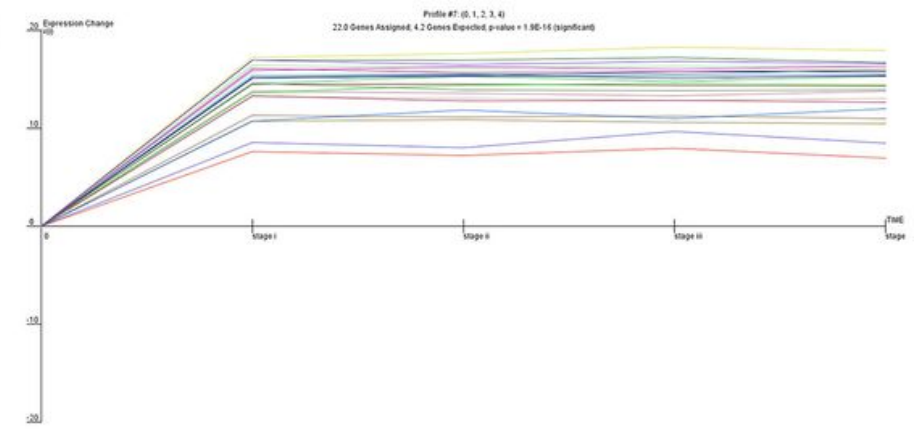

D

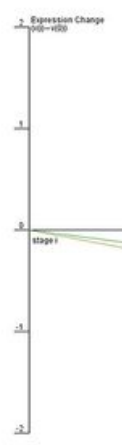

C
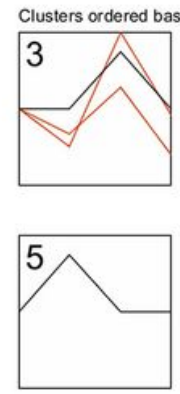
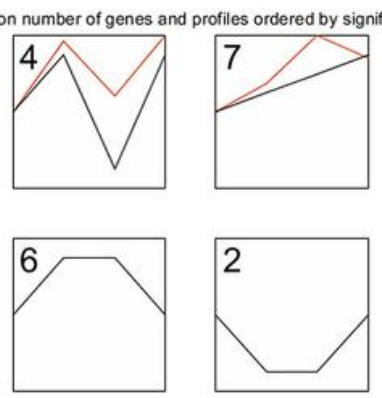
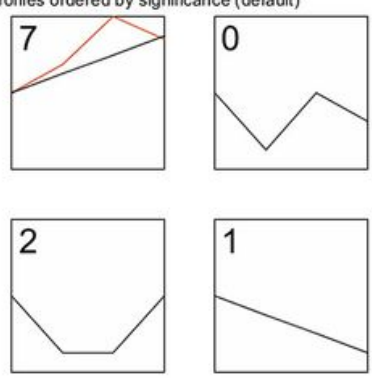

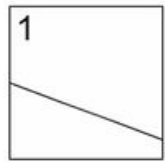




\section{Figure 7}

Identification of trend-related SNHGs To obtain the trend-related SNHGs based on the classification of tumor stage diagnosis, including stage I, stage II, stage III, and stage IV, the data was processed using STEM software for trend analysis. First, the data was processed with 'No normalization/add 0 ' and screened with $p<0.05$. Then the data was processed according to the parameter 'normalize data' and screened with $p<0.1$. According to the single module, there was no obvious change trend among the various stages. Following amplification, the expression of each gene is shown according to the stage node. Two SNHGs, including SNHG23 and SNHG24, have obvious trend changes. A: The trend analysis of 'No normalization/add 0'. The colored module in the figure represents significant trend with $p<$ 0.05. Each colored line represents the expression trend of a gene at different points, and the black line represents the general trend of the change of these genes. The colorless modules represent non-significant trends and were excluded from the subsequent analyses. B: The trend analysis of 'normalize' module with $p<0.05$. There are no colored modules. C: The results of 'No normalize/add 0' module. A single module in the sample graph of trend analysis is magnified to show the expression of each gene according to the stage node. D: The results of 'normalize' single module.

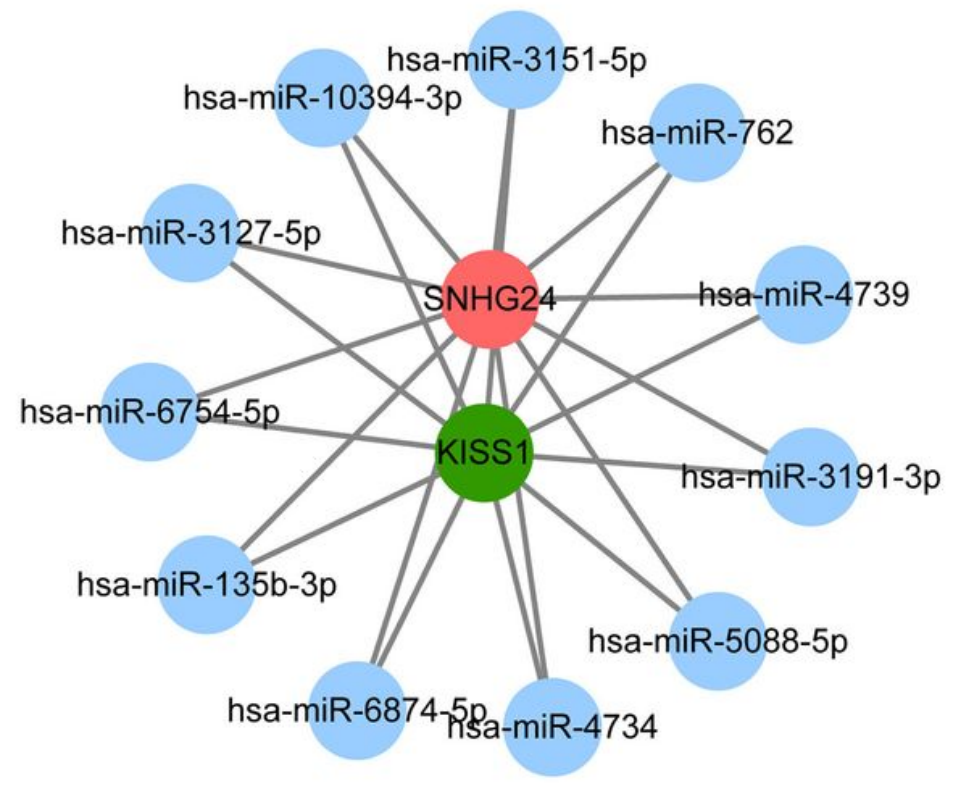

\section{Figure 8}

Construction of ceRNA network To identify the ceRNA interaction pairs, the interaction pairs of IncRNA-mRNA, IncRNA-miRNA, and mRNA-miRNA were integrated to construct the ceRNA network. The ceRNA network was constructed with 11 miRNAs, one IncRNA and one mRNA. The red circle represents SNHGs, the green circle represents $\mathrm{mRNA}$, and the blue circle represents miRNA. 


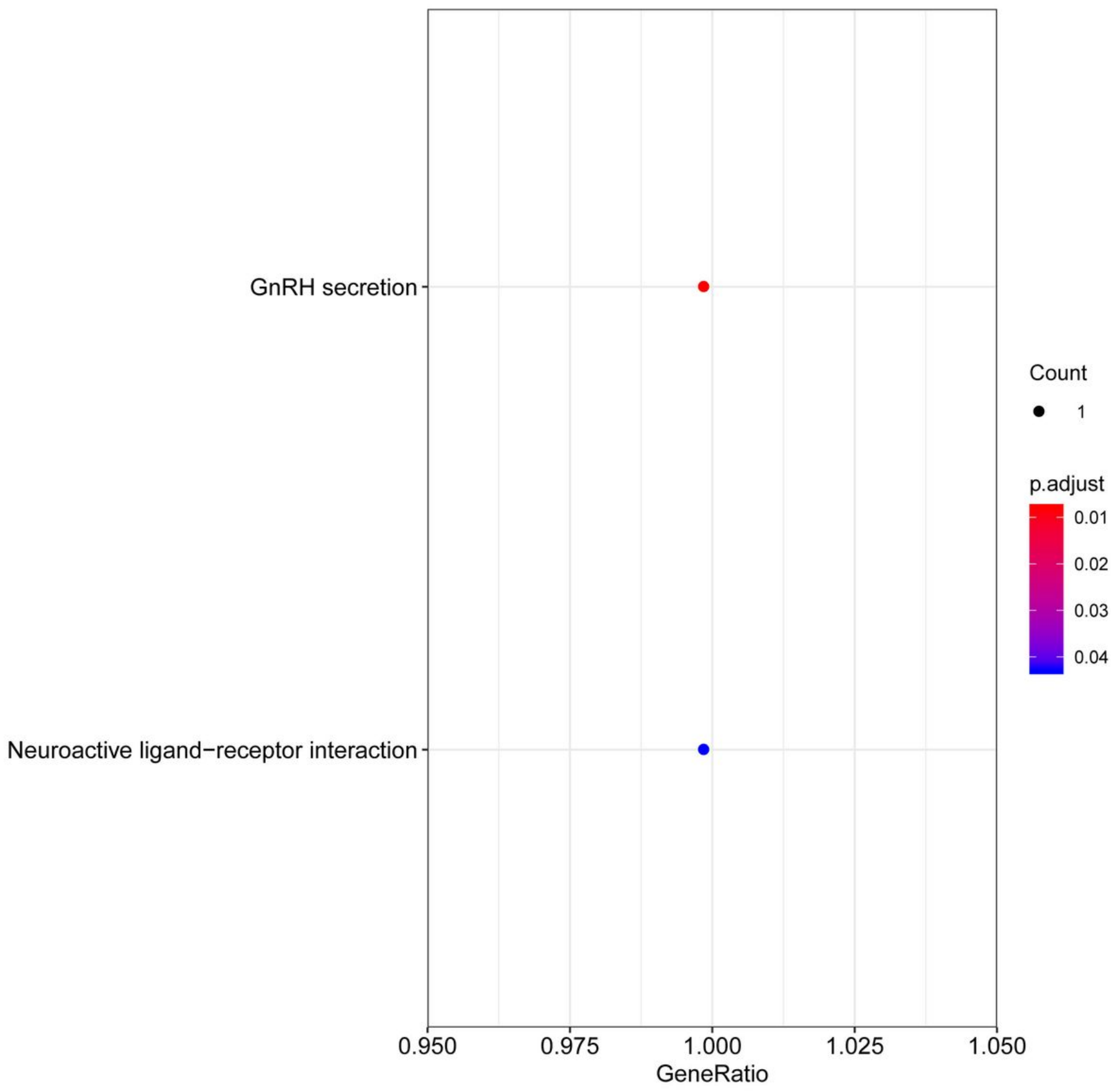

\section{Figure 9}

Gene ontology (GO) functional and pathway enrichment analysis in ceRNA network To identify the pathways that might be affected by the SNHGs, the genes in the ceRNA network were subjected to KEGG pathway annotation using the clusterprofiler tool. The results revealed that SNHG24 is mainly correlated with GnRH secretion and neuroactive ligand-receptor interaction in pancreatic cancer.

\section{Supplementary Files}

This is a list of supplementary files associated with this preprint. Click to download. 
- Supplementaryfiles1.docx

Page 38/38 